\title{
Application of Airy beam light sheet microscopy to examine early neurodevelopmental structures in 3D hiPSC-derived human cortical spheroids
}

Dwaipayan Adhya ${ }^{1,2+}$, George Chennell ${ }^{1 \dagger}$, James A. Crowe ${ }^{1,3 \dagger}$, Eva P. Valencia-Alarcón ${ }^{1,3}$, James Seyforth ${ }^{4}$, Neveen A. Hosny ${ }^{4}$, Marina V. Yasvoina ${ }^{5}$, Robert Forster ${ }^{4}$, Simon Baron-Cohen ${ }^{2}$, Anthony C. Vernon ${ }^{1,3}$ and Deepak P. Srivastava ${ }^{3^{*}}$ (1)

\begin{abstract}
Background: The inability to observe relevant biological processes in vivo significantly restricts human neurodevelopmental research. Advances in appropriate in vitro model systems, including patient-specific human brain organoids and human cortical spheroids (hCSs), offer a pragmatic solution to this issue. In particular, hCSs are an accessible method for generating homogenous organoids of dorsal telencephalic fate, which recapitulate key aspects of human corticogenesis, including the formation of neural rosettes - in vitro correlates of the neural tube. These neurogenic niches give rise to neural progenitors that subsequently differentiate into neurons. Studies differentiating induced pluripotent stem cells (hiPSCs) in 2D have linked atypical formation of neural rosettes with neurodevelopmental disorders such as autism spectrum conditions. Thus far, however, conventional methods of tissue preparation in this field limit the ability to image these structures in three-dimensions within intact hCS or other 3D preparations. To overcome this limitation, we have sought to optimise a methodological approach to process hCSs to maximise the utility of a novel Airy-beam light sheet microscope (ALSM) to acquire high resolution volumetric images of internal structures within hCS representative of early developmental time points.
\end{abstract}

Results: Conventional approaches to imaging hCS by confocal microscopy were limited in their ability to image effectively into intact spheroids. Conversely, volumetric acquisition by ALSM offered superior imaging through intact, non-clarified, in vitro tissues, in both speed and resolution when compared to conventional confocal imaging systems. Furthermore, optimised immunohistochemistry and optical clearing of hCSs afforded improved imaging at depth. This permitted visualization of the morphology of the inner lumen of neural rosettes.

Conclusion: We present an optimized methodology that takes advantage of an ALSM system that can rapidly image intact 3D brain organoids at high resolution while retaining a large field of view. This imaging modality can be applied to both non-cleared and cleared in vitro human brain spheroids derived from hiPSCs for precise examination of their internal 3D structures. This process represents a rapid, highly efficient method to examine and quantify in 3D

\footnotetext{
*Correspondence: deepak.srivastava@kcl.ac.uk

${ }^{\dagger}$ Dwaipayan Adhya, George Chennell and James A. Crowe contributed equally to this work

${ }^{3}$ MRC Centre for Neurodevelopmental Disorders, King's College London, London, UK

Full list of author information is available at the end of the article
} permits use, sharing, adaptation, distribution and reproduction in any medium or format, as long as you give appropriate credit to the original author(s) and the source, provide a link to the Creative Commons licence, and indicate if changes were made. The images or other third party material in this article are included in the article's Creative Commons licence, unless indicated otherwise in a credit line to the material. If material is not included in the article's Creative Commons licence and your intended use is not permitted by statutory regulation or exceeds the permitted use, you will need to obtain permission directly from the copyright holder. To view a copy of this licence, visit http://creativecommons.org/licenses/by/4.0/. The Creative Commons Public Domain Dedication waiver (http://creativeco mmons.org/publicdomain/zero/1.0/) applies to the data made available in this article, unless otherwise stated in a credit line to the data. 
the formation of key structures required for the coordination of neurodevelopmental processes in both health and disease states. We posit that this approach would facilitate investigation of human neurodevelopmental processes in vitro.

\section{Introduction}

Human brain organoids represent a revolutionary step forward in our ability to investigate human neurodevelopment through the use of patient-specific human induced pluripotent stem cells (hiPSC) to generate neural tissues [1-3]. Brain organoids recapitulate a more native state of neurodevelopment and thus have enabled the in vitro study of molecular and cellular events during early human neurodevelopment [4-7]. Early development is also emerging as a key period for the emergence of atypical cellular and molecular processes thought to contribute to the likelihood of neurodevelopmental disorders such as autism spectrum conditions (here after referred to as autism) [8-12]. Differentiation of hiPSCs towards a forebrain neuronal linage has been shown to recapitulate many of the hallmarks of early neurodevelopment. Exposure of hiPSCs to dual SMAD inhibition results in first the generation of neuroepithelium cells that subsequently differentiate into neural progenitor cells (NPCs) [13-15]. These cells then self-organise around a central lumen: a structure called a 'neural rosette' $[2,16]$. These neural rosettes display apical-basal polarity and are thought to represent the formation of the neural tube during early brain development $[2,16,17]$. Formation of neural rosettes is crucial for neural development, and perturbations in the development of these structures can result in premature or abnormal neurogenesis and astrogenesis [18]. Several studies using patient-derived hiPSCs or human embryonic stem cells (hESCs) have linked abnormal neural rosette development and subsequent neurogenesis with the pathophysiology of neurodevelopmental disorders. For example, NPCs differentiated from hESCs generated from individuals with Fragile $\mathrm{X}$ syndrome, a syndromic form of autism, developed neural rosettes significantly slower than control hESCs and displayed reduced neurogenic potential [19]. Biallelic deletion of TSC2, another syndromic form of autism, led to hyperfunction of mTORC1 in neural rosettes and subsequently, impaired neural differentiation as well as increased astroglial differentiation [20]. Furthermore, our group has recently observed anomalies in neural rosette formation between typical and autistic hiPSCs, which appear to have long-term consequences in neural development [12]. While these studies have predominately used 2D differentiation protocols, these abnormalities in neural rosette formation were also recapitulated in 3D preparations [12]. Thus, brain organoids offer a unique opportunity to study pathophysiological mechanisms thought to occur in these disorders $[9-12,21]$.

Several different methods have been used to generate 3D brain organoids from hiPSCs that recapitulate the complex cellular developmental processes involved in the generation of specific brain regions [1-3, 22-24]. One example is the generation of human cortical spheroids (hCSs), which generate dorsal cortical cells [2]. In this method, the NPCs that self-organise around the central lumen of a neural rosette; these structures also display apical-basal polarity, giving rise to post-mitotic neurons similar to those found within the forebrain $[2,16,17]$. Thus, brain organoids like hCSs provide a $3 \mathrm{D}$ in vitro system in which mechanisms relevant for human neurodevelopment and disorders such as autism can be studied.

At present, microscopic analysis of human brain organoids is primarily undertaken by conventional methodologies such as confocal microscopy, which require deconstruction of the 3D structure [1, 2, 23]. This approach typically involves imaging of $2 \mathrm{D}$ tissue slices of 3D organoids. This results in a loss of the overall 3D tissue-like structural fidelity, and thus, the phenotypic resemblance to their original in vivo correlates. Hence, the significance of understanding cellular processes, organisation and complexity within a system that mimics native human tissue in a 3D state is lost [25]. To image intact nervous tissues, high-speed, high-resolution volumetric imaging methods are required over large fieldsof-view. New imaging modalities such as, light sheet microscopy (LSM) are rapidly gaining importance for imaging intact biological specimens [26, 27]. The advantage of using LSM over confocal microscopy is that it rapidly $(500 \times$ faster) produces $3 \mathrm{D}$ volumetric images and due to the speed and the specificity of planar illumination of light sheet when compared with whole-sample illumination using confocal microscopy [28]. These factors reduce the dwell time on each plane imaged producing less photo-damage to the specimen. However, two important factors need to be taken into consideration when utilizing this approach: (1) due to lipid content, there is considerable loss of excitation signal strength and scattering of emission light in imaging of large volumes of tissue that may result in a rapid degradation of signal with increasing depth of tissue; and (2) antibodies are often accumulate in superficial regions of tissue, due 


\begin{abstract}
(See figure on next page.)
Fig. 1 Setup and resolution of Airy-beam light sheet microscopy (ALSM). a ALSM system is equipped with 2 water dipping objectives-10 $\times 0.3$ NA illumination and 20X 0.5 NA detection objective - positioned at a $45^{\circ}$ angle. Biological specimens are placed on the X-Y-Z stage. Movement of the stage is controlled by a Z-axis Stepper motor (ZSM) which moves the sample along the optical axis of the detection objective. The system is equipped with $405 \mathrm{~nm}, 488 \mathrm{~nm}, 561 \mathrm{~nm}$ and $640 \mathrm{~nm}$ wavelength lasers. b Illumination objective focuses the Airy-beam (green) on to sample and the detection objective captures the emission (yellow) at 90 degrees. The geometry of the objectives gives the system a (hypothetical) working distance of $3.5 \mathrm{~mm}$. c An example of the Airy-beam produced by focusing the $488 \mathrm{~nm}$ laser though water. $\mathbf{d}$ Representative image of a $500 \mathrm{~nm}$ Tetraspeck fluorescent beads imaged at 405, 488, 561 and 640 wavelengths; image has not undergone deconvolution. Characteristic Airy lobes can be seen in the axial plane. e Deconvolved image of the fluorescent bead in C). Axial Airy lobes have been removed following deconvolution. $\mathbf{f}$ Image of a single $500 \mathrm{~nm}$ Tetraspeck fluorescent bead; the PSF of a single $500 \mathrm{~nm}$ bead is $0.625 \mu \mathrm{m}$ diameter at full width at half maximum (FWHM) when imaged at $488 \mathrm{~nm}$. Scale bars $=500 \mathrm{~nm}$ unless stated otherwise
\end{abstract}

to reduced penetrance of antibodies or permeabilising agents deep into tissue, thus reducing visibility of target proteins in deeper sections of the tissue.

Several methods are now available to render intact tissues optically transparent [29-32]. These aqueous clearing techniques reduce light scattering by removing tissue lipids and replacing them with a clear matrix. As reported by $\mathrm{Xu}$ et al. [32], replacing lipids with a clear matrix facilitates greater penetration of antibodies. The combined effect enables significant preservation of both tissue ultrastructure and the accessibility of native proteins to antibody probes $[29,31,32]$. To date, only a limited number of studies have applied the use of LSM or tissue clearing in combination with human brain organoids. For example, Renner and colleagues used cleared cerebral organoids to visualise the organisation of ventricles and connectivity between different regions within cerebral organoids [33]. However, in this study, the authors utilized conventional imaging approaches that require the manual reconstruction of 3D structures from 2D images, which is both time-consuming and may result in the loss of the inherent complexities of the biological specimen [25]. In a different study, Li and colleagues used LSM to examine cortical folding in cerebral organoids [34], but not to examine the organisation of cellular structures internally. To our knowledge, few if any studies have used a combination of LSM with tissue clearing to examine the organisation of internal cellular structures in human brain organoids, despite current interest in these technologies [25, 28].

In this study, we have: (1) compared conventional tissue processing and image acquisition methods (confocal microscopy) against a novel methodology of presenting intact 3D tissue for volumetric acquisition using LSM; and (2) optimised immunohistochemical approaches in combination with tissue clearing to improve acquisition of neural rosettes structures at depths greater than $500 \mu \mathrm{m}$ deep within hCSs. Specifically, we imaged intact hCSs generated from hiPSCs, using an Airy-beam light sheet microscopy (ALSM) system [27], in combination with an optimized immunohistochemical protocol based on the Fast free-of-acrylamide clearing tissue (FACT) methodology [32] and a refractive index (RI)-matched fast tissue clearing approach based on the ScaleS [31]. We confirm that the ALSM is superior in imaging through intact, non-clarified, in vitro tissues, both in speed and resolution as compared to conventional confocal imaging systems. Furthermore, optimised immunohistochemistry and clearing of hCSs allowed for enhanced visualisation of internal structures. This enabled the visualisation of the inner lumen of neural rosettes in 3D and indicated that these structures may develop as a polarised tubelike structure. These finding indicate that ALSM imaging in combination with optimized immunohistochemistry and tissue clearing promises to yield significantly better $3 \mathrm{D}$ imaging of internal structures in brain organoids and thus will facilitate the study of neurodevelopmental processes relevant for typical and atypical development.

\section{Results}

\section{Application of Airy-beam light sheet microscopy (ALSM) with high resolution}

A particular advantage of ALSM is that it has a wider field of view (FOV) than Gaussian or Bessel-based light sheet beams due to the properties of the propagationinvariant Airy beam [27] (Fig. 1a-c). This in-turn means that the high axial resolution is maintained across that FOV without the need for compromising the FOV or resolution, unlike other light sheet systems. The Airy-beam delivers a field of view 20 times larger than a Gaussian beam and 8 times larger than a Bessel beam equipped on the same system [27]. The Airy-beam also provides an axial resolution comparable to that of the Gaussian and twice as good as the Bessel beam [27]. The Airy-beam's characteristic asymmetric excitation pattern creates lobes spreading the beam across the FOV, lowering the overall light exposure to the sample resulting in $80 \%$ less photo-bleaching in comparison with the Gaussian beam [27]. Furthermore, it only requires a single exposure per $\mathrm{z}$ slice compared to the multiple acquisitions required for some light sheet approaches such as dual inverted selective plane illumination microscopy, further reducing 
a

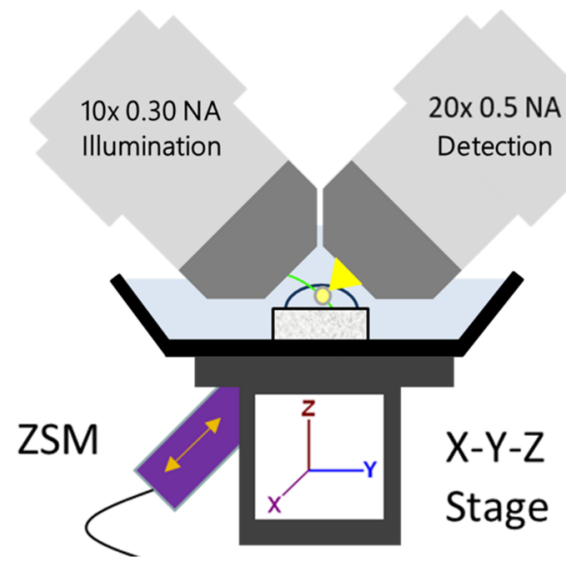

C

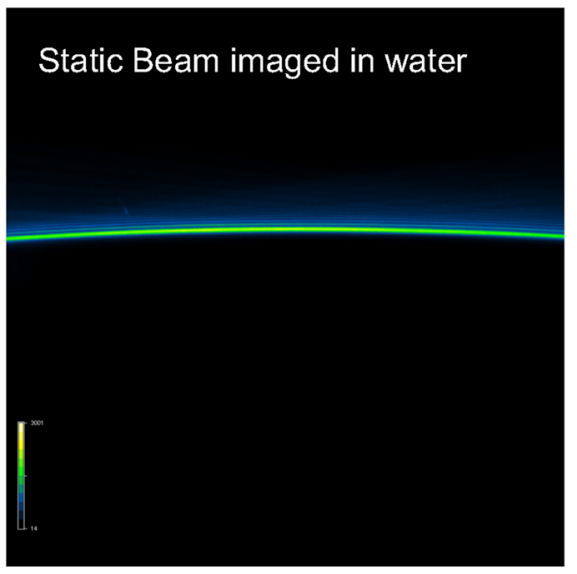

e

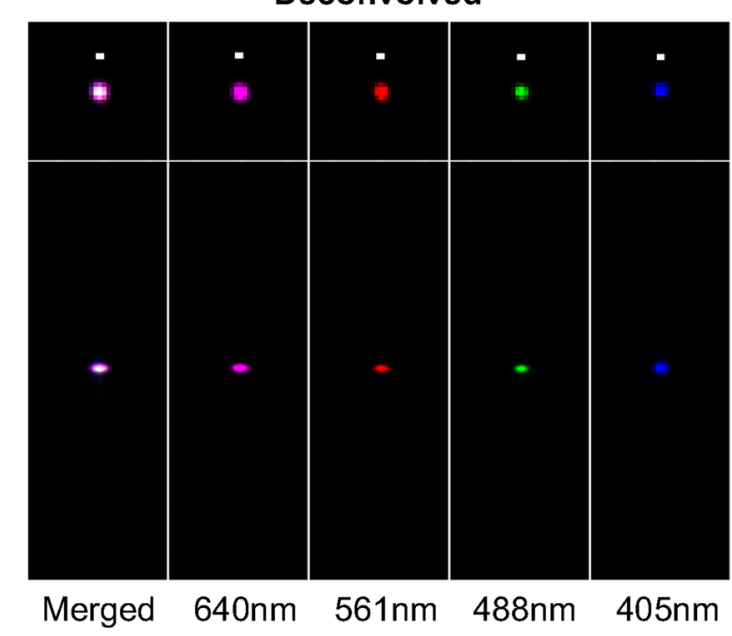

b

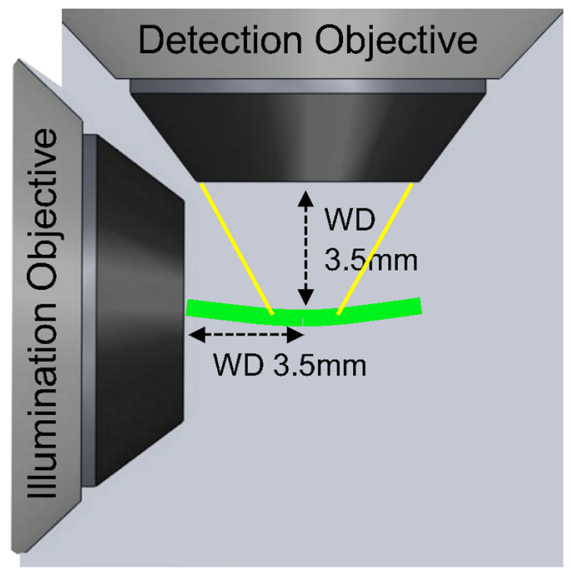

d

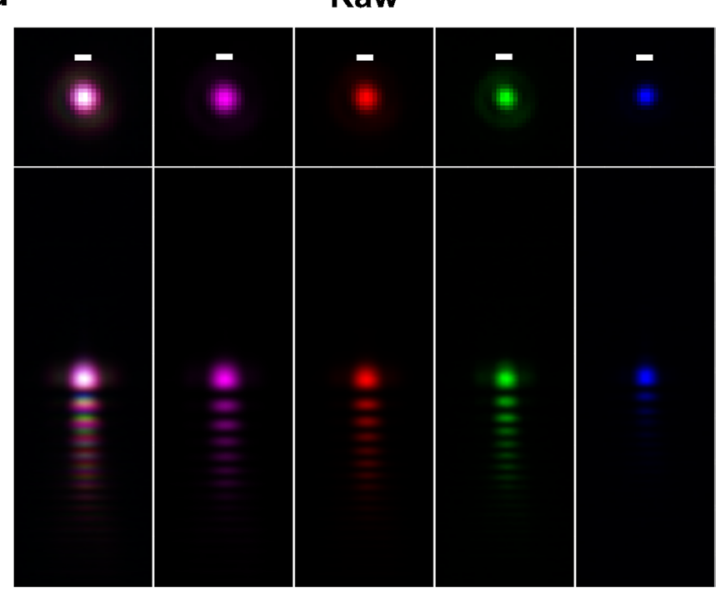

Merged $\quad 640 \mathrm{~nm} \quad 561 \mathrm{~nm} \quad 488 \mathrm{~nm} \quad 405 \mathrm{~nm}$
$625 \mathrm{~nm}$

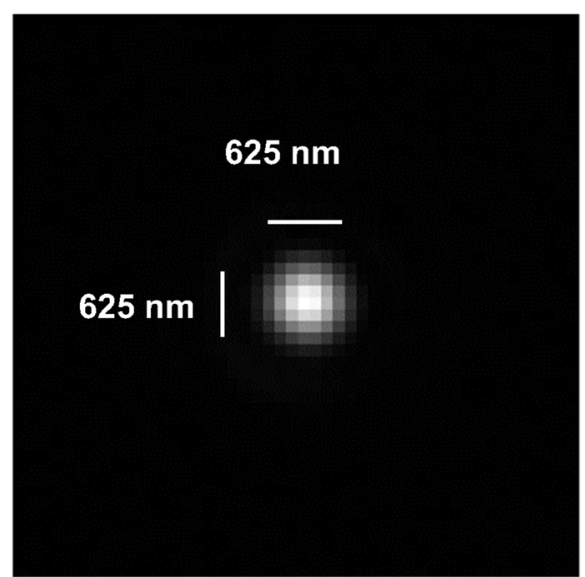




\section{(See figure on next page.)}

Fig. 2 Confocal imaging of wholemount day 7 human cortical spheroids (hCSs) is limited at depth. a Representative confocal images of intact (wholemount) day 7 hCS showing strong immunostaining for the neuroepithelial/NPC markers Nestin and Sox2. b Representative confocal image of wholemount day $7 \mathrm{hCS}$ demonstrates hallmarks of the emergence of neural rosettes, as determined by N-cadherin ( $\mathrm{N}$-cad) staining of apical membrane and surrounding Pax6-positive cells. c Maximally projected and orthogonal views of Z stacks of intact Day 7 hCSs acquired by confocal imaging. Confocal imaging displays a drop-off in fluorescence intensity correlated with acquisition depth (Z planes). (i) DAPI, a non-antibody stain for DNA shows incomplete detection throughout the tissue-like structure. Similarly, (ii) Nestin-positive and (iii) Sox2-positive immunostaining displays even greater drop off in depth-detection, shown in (iii) as a fluorescence-intensity heatmap. $\mathbf{d}$ Graphic depicting changes in fluorescent intensity (A.U.) with increasing imaging depth $(\mu \mathrm{m})$ of Ci-iii. Scale bars $=50 \mu \mathrm{m}$

photo-toxicity [27]. Thus, using an exposure time of $50 \mathrm{~ms}$ (ms), the acquisitions of a 1500 slice stack would take $75 \mathrm{~s}$ per channel. The ALSM system uses 2 water dipping objectives. The geometry of the objectives means the maximum sample area the light sheet can image is approximately $3.5 \mathrm{~mm}$ in depth ( $X Z$ axis) and $3.5 \mathrm{~mm}$ in width ( $X$ axis), but along the $y$-axis it is possible to have a dimension larger than $3.5 \mathrm{~mm}$ (Fig. 1a, b). However, due to the physical dimensions of the space in between the lenses and the need to move the sample around to acquire an image, the actual FOV is $600 \times 600 \times 600 \mu \mathrm{m}$.

The resolution of the ALSM system used in this study was determined by measuring $500 \mathrm{~nm}$ sub-resolution Tetraspeck fluorescent beads at multiple wavelengths. Raw (pre-deconvolution) image acquisition of beads resulted in a data stack that upon re-slicing displayed Airy lobes artefacts in the axial plane (Fig. 1d). All bead stacks were subsequently deconvolved [27] using an extracted bead PSF and processed using RichardsonLucy deconvolution (MSquared Cubes software). This restored the image, increasing the signal-to-noise and removed the geometric curvature of the Airy-beam (Fig. 1e). Measurements of resolution was assessed by determining the "Full width at half maximum" (FWHM) at the $488 \mathrm{~nm}$ wavelength of resliced beads where the mean system has a resolution of $0.625 \mu \mathrm{m}$ (Fig. 1f).

\section{Challenges associated with imaging of hCSs using confocal microscopy}

In order for us to assess the utility of ALSM for the imaging of 3D organoid cultures, we differentiated 3 well characterised hiPSC lines from our group [12, 15] using a free-floating directed culture method to generate 3D cortical spheroids (hCSs) [2] (Additional file 1: Figure $1 \mathrm{~A}-\mathrm{E})$. At day 7 , hCS specimens were $\sim 100-300 \mu \mathrm{m}$ in size (Additional file 1: Figure 1C). Cryo-sectioning of day $7 \mathrm{hCS}$ often resulted in tissue damage. Therefore, day $7 \mathrm{hCSs}$ were imaged as 'wholemount' intact samples by conventional confocal microscopy. As expected hCS generated from hiPSCs demonstrated the key hallmarks of in vitro cortical differentiation $[2,6,16]$. At this time point, hCSs were positive for the early neuroepithelium markers Nestin and Sox2 (Fig. 2a). These spheroids also displayed N-cadherin-positive membranes (Fig. 2b), indicative of the emergence of neural rosettes $[6,16]$. In addition, hCSs were generally positive for Pax6, reinforcing the fate specification of a cortical neural progenitor cell (NPC) population (Fig. 2b).

Although confocal microscopy was easily able to acquire z-stack images of superficial regions of hCSs, it was insufficient to acquire images of the entire spheroids. On average, we were only able to image up to 50-100 $\mu \mathrm{m}$ deep into hCS before significant signal dropoff occurred. This is demonstrated in Fig. 2c where we have imaged a day $7 \mathrm{hCS}$ of approximately $300 \mu \mathrm{m}$ in diameter, stained for DAPI, Nestin and Sox2. Confocal images were acquired as a z-stack-images were acquired every $20 \mu \mathrm{m}$ ( $1 \mathrm{z}$ step depth $=0.4 \mu \mathrm{m}$, using a $63 \times$ NA1.4 oil immersion objective) resulting in a total imaged depth of $280 \mu \mathrm{m}$ (Additional file 1: Figure 2A-C). In all 3 channels, fluorescent signal towards the centre of the hCS tissue weakened dramatically at greater depth ( $>100 \mu \mathrm{m}$ ), as demonstrated by intensity profiles for each channel through the z-plane (Fig. 2c, d; Additional file 1: Figure 2D). This indicated either a poor acquisition of fluorescent signal via confocal acquisition or a failure of antibodies to fully permeate the tissue during preparation. However, fluorescent signal was similarly weakened beyond $100 \mu \mathrm{m}$ in the DAPI channel, which is a highly penetrant small molecule fluorescent stain for DNA (Fig. 2c, d; Additional file 1: Figure 2D). This indicated that confocal microscopy was unable to image internal structures within intact spheroids.

We next characterised day $17 \mathrm{hCS}$, which were considerably larger than day $7 \mathrm{hCS}$. As day $17 \mathrm{hCSs}$ ranged between 800 and $1200 \mu \mathrm{m}$ in diameter (Additional file 1: Figure $1 \mathrm{C}$ ), it was not possible to image intact day 17 spheroids using confocal microscopy. Consequently, day $17 \mathrm{hCSs}$ were prepared as 20 or $60 \mu \mathrm{m}$ cryo-sections before immunostaining and subsequent imaging with by confocal microscopy. Imaging of thin $(20 \mu \mathrm{m})$ day $17 \mathrm{hCS}$ cryo-sections demonstrated the expected neural rosette organisation of axial polarity with a lumen delineated by adjoining neural progenitor cells (Fig. $3 a-d)$. The inner lumen of neural rosettes was highlighted by the presence of tight junction proteins $\mathrm{ZO}-1$ and $\mathrm{N}$-cadherin ( $\mathrm{N}$-cad), 

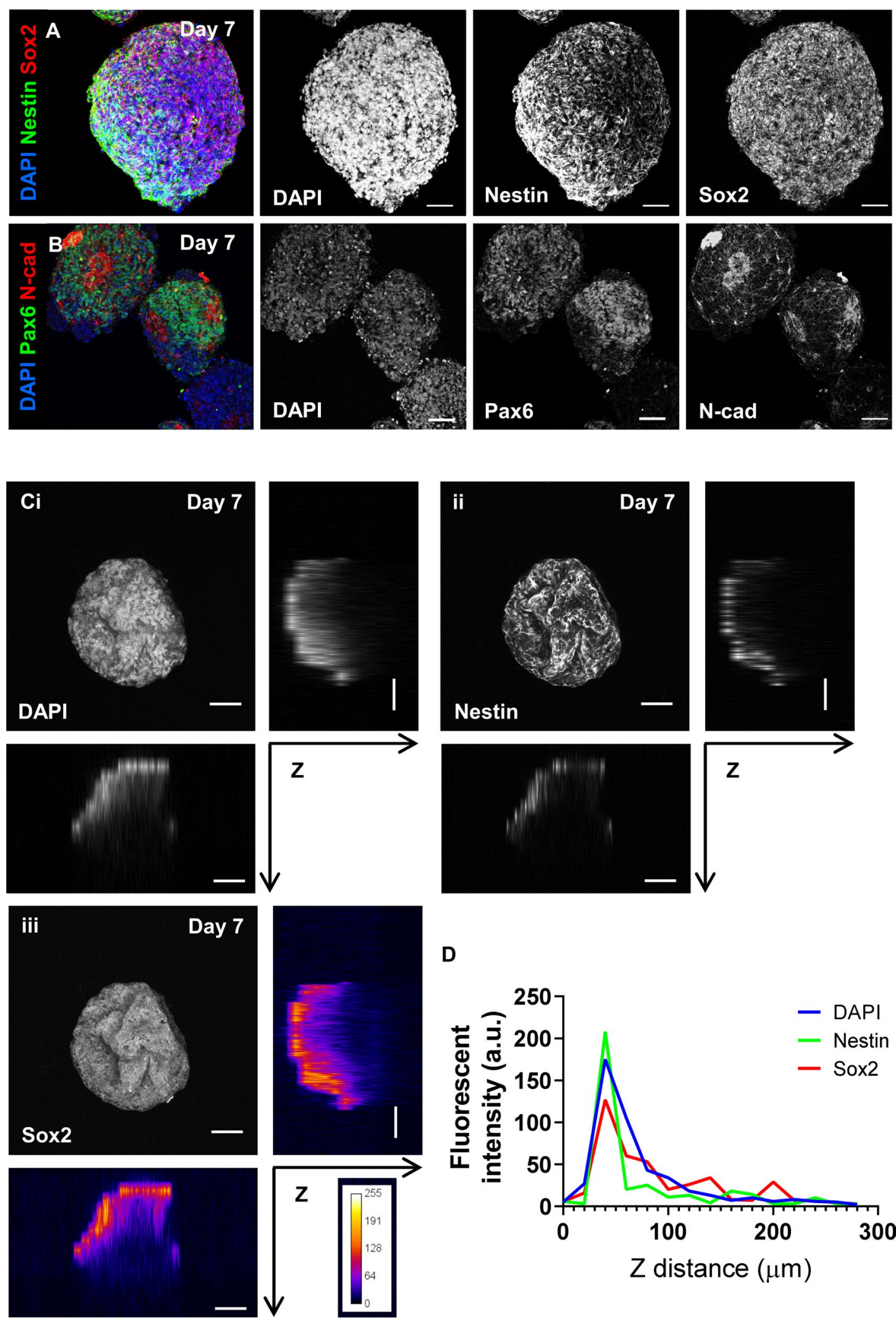

D

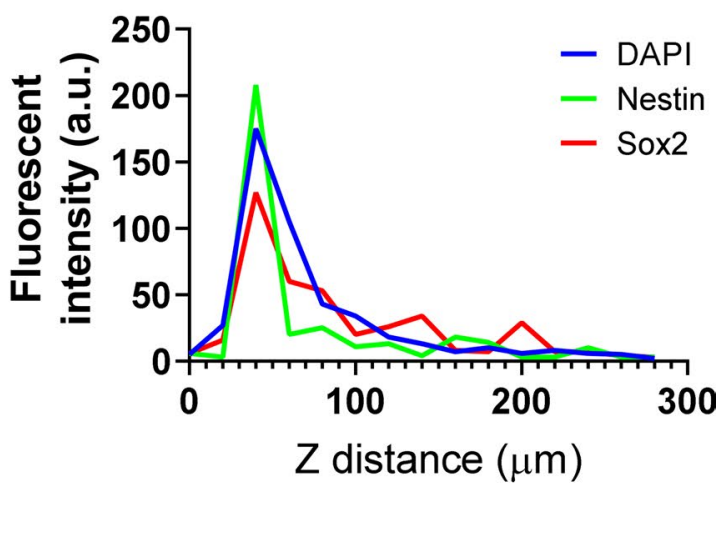




\section{(See figure on next page.)}

Fig. 3 Confocal imaging of cryo-sectioned day 17 hCSs reveals incomplete acquisition of neural rosette 3D morphology. a-d Representative confocal images of sectioned $(20 \mu \mathrm{m})$ day $17 \mathrm{hCSs}$; a ZO-1 staining of apical membrane is surrounded by apico-basal polarised Nestin filaments; b Pax6-positive cells self-organise around N-cad positive foci; c Immature DCX-positive neuronal processes are found outside of neural rosette structures; and $\mathbf{d}$ Map2-positive neuronal processes are also found to surround Pax6-positive NPCs organised into rosettes. e Confocal imaging of $60 \mu \mathrm{m}$ sectioned day $17 \mathrm{hCS}$ express signs of cortical rosette formation. Newly formed PKC $\lambda$-positive membranes are seen at the edge of the tissue as indicated by yellow arrows. W Within thicker sections $(60 \mu \mathrm{m})$, internalised tubular structures positive for $\mathrm{N}$-cad are observed, suggesting that the inner lumen of neural rosettes may form into tube-like structures. $\mathbf{g}$ Phalloidin 488-positive actin filaments (RDYPROBE) show a concentrated organisation around ventricle-like structures. Magenta arrows in $\mathbf{e}-\mathbf{g}$ images highlight the ovoid formation of cortical rosettes with a pinched end, whereas blue arrows highlight formation of a tubular-like structure. Scale bars $=100 \mu \mathrm{m}(\mathbf{a}-\mathbf{d}) ; 50 \mu \mathrm{m}(\mathbf{e}-\mathbf{g})$

as well as PKC $\lambda$-positive cells, indicating maintenance of adherent junctions by neural progenitors (Fig. 3a-c). In the region immediately surrounding the inner lumen, nestin- and Pax6-positive cells were radially organised as expected for early NPCs (Fig. 3a, b). Consistent with our recent study [12], hCSs generated from autism hiPSCs displayed aberrant neural rosette formation, as demonstrated by incomplete ZO-1 apical membrane formation (Additional file 1: Figure 3B). Furthermore, the radial organisation of Pax6-positive NPCs around ZO-1-positive lumens was disrupted in autism hCSs compared to control hCSs (Additional file 1: Figure 3A and B). DCX and MAP2-positive cells representative of the differentiation of NPCs into newborn and immature neuronal cells could be seen constituting the outer layers of the neural rosette (Fig. 3c, d). Overall, the description of neural rosette organisation and structure was similar to that previously described in 3D studies $[2,6,16]$.

Interestingly, imaging of thicker sections $(60 \mu \mathrm{m})$ occasionally revealed further insight into the emergence of rosette structures as well as greater detail on the potential morphology of the rosette lumen. For example, PKC $\lambda$, a putative organisational marker of neural rosettes, could at times be seen in pinched groups of cells at the outer layer of tissue (Fig. 3e; yellow arrows). These PKC $\lambda$-positive cells are thought to represent cells that internalise to subsequently form the inner lumen of rosettes $[16,35]$ In addition, when we imaged thick $(60 \mu \mathrm{m})$ sections stained for N-cadherin, we were able to occasionally observe rosette lumens as uneven, elongated or tubular structures (Fig. 3f). This raised the possibility that the inner lumen of neural rosettes develops a tube-like morphology when spatially un-restricted in 3D and that we had serendipitously sectioned laterally through the inner lumen of a rosette (Fig. 3f; cyan arrow). A similar morphology was also observed in hCS sections stained for F-actin using an AlexaFluor 488-conjugated phalloidin stain (ActinGreen $^{\mathrm{TM}} 488$ ReadyProbes $^{\mathrm{TM}}$-referred to as RDYPROBE). RDYPROBE staining of F-actin, a major cytoskeletal component, was concentrated around the inner lumen of neural rosettes (Fig. 3g; cyan arrow) similar to previous reports $[16,18]$. However, F-actin staining could also be seen as polarised tube-like structures (Fig. 3g; magenta arrows) similar to that seen with $\mathrm{N}$-cadherin staining. Taken together, these data indicate that rosette lumens may develop into tube-like structures when grown in 3D, and that cryo-sectioning followed by confocal imaging is not sufficient to fully capture the morphology of these structures in sectioned spheroids.

\section{ALSM imaging of intact hCS}

Having established that confocal microscopy was limited to imaging $\sim 100 \mu \mathrm{m}$ deep into intact spheroids, we next tested the ability of ALSM to image at greater depths. To this end, we generated day $30 \mathrm{hCSs}$ and stained them with RDYPROBE to label F-actin, and thus the structure of neural rosettes. An ALSM image of a day 30 hCSs (diameter $=\sim 1.2 \mathrm{~mm}$ ) was acquired as a single $\mathrm{z}$-stack. A raw output max intensity Z-projection (z-stack $=500$ images $-600 \times 600 \times 200 \mu \mathrm{m})$ can been seen in Additional 1 file 1: Figure 4Ai (Raw image). This image stack subsequently underwent deconvolution using a Richardson-Lucy algorithm [36, 37] specific to the ALSM (MSquared Cubes software). Following this image processing, multiple neural rosettes with tube-like structures could be observed within the hCS (Additional file 1: Figure 4Aii-Deconvolved Image). However, we noted that some non-specific staining on the surface appeared very bright leading to features of interest appearing dimmer. Therefore, we removed the images of the outer layer of tissues from the 3D image stack. This removed highly stained cells at the edge which had a higher fluorescence than internal structures and thus allowed for a clearer visualisation of internal rosettes (Additional file 1: Figure 4B). Multiple neural rosette lumens with different shapes and sizes could be observed in both 3D renders and max projection images (Fig. 4a, b). Larger rounder rosette lumens were observed towards the middle of the tissue, whereas smaller extended tube-like rosettes can be found towards the edge of the tissue (Fig. 4a, b). These structures had diameters ranging between 20-50 $\mu \mathrm{m}$, and lengths between $20-200 \mu \mathrm{m}$.

We next imaged a day $30 \mathrm{hCS}$ that was stained with RDYPROBE and immunostained with the NPC marker 

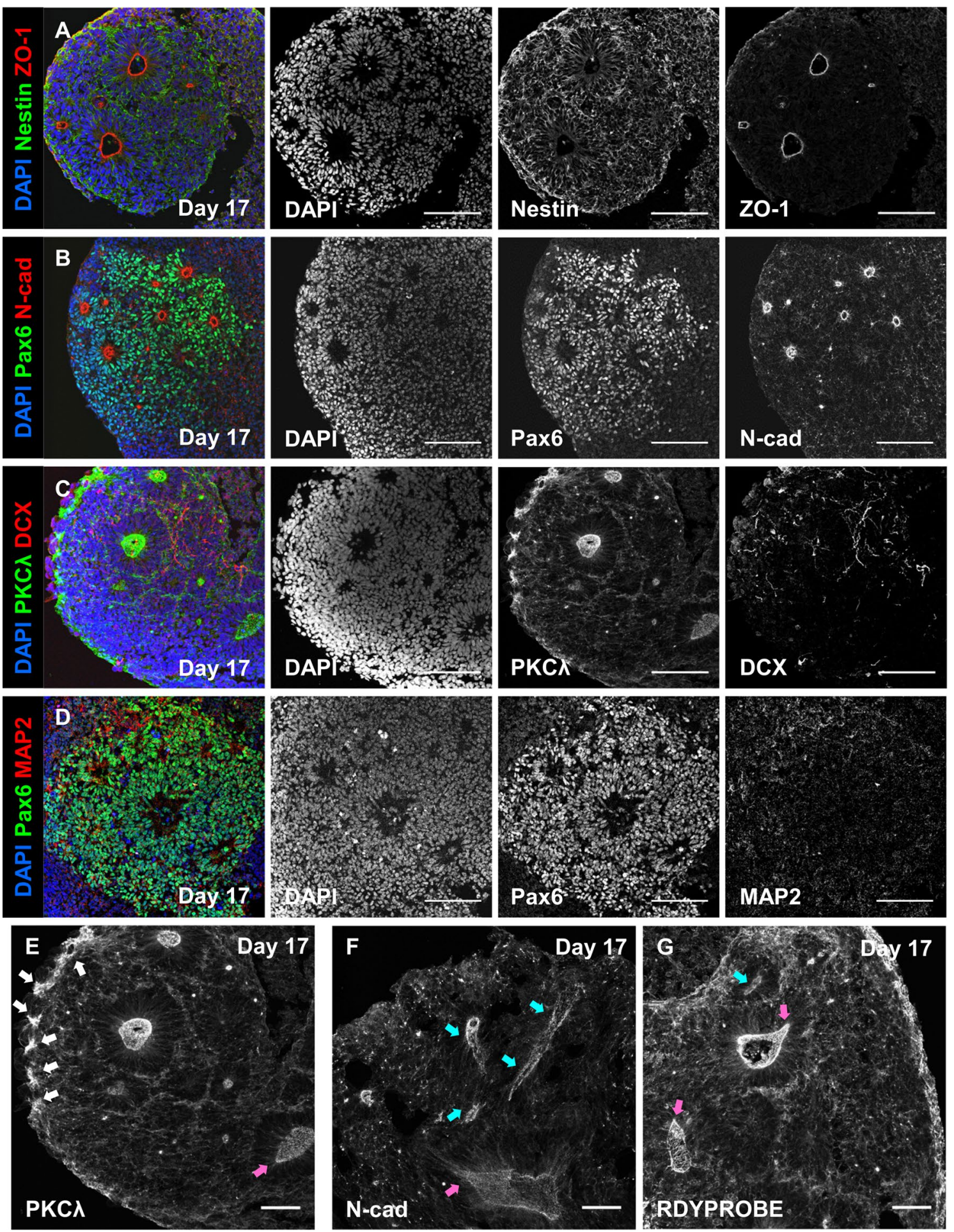

Nestin to assess signal intensity deep within day $30 \mathrm{hCSs}$ (Fig. 4c and Additional file 1: Figure 4C). As expected, F-actin staining revealed several neural rosette lumens within the spheroid. High magnification zoom-ins of
F-actin enriched apical membrane showed that Nestinpositive cells were organised radially around the central lumen (Fig. 4c) with Nestin filaments being polarised and projecting away from the central lumen (Fig. 4c). 

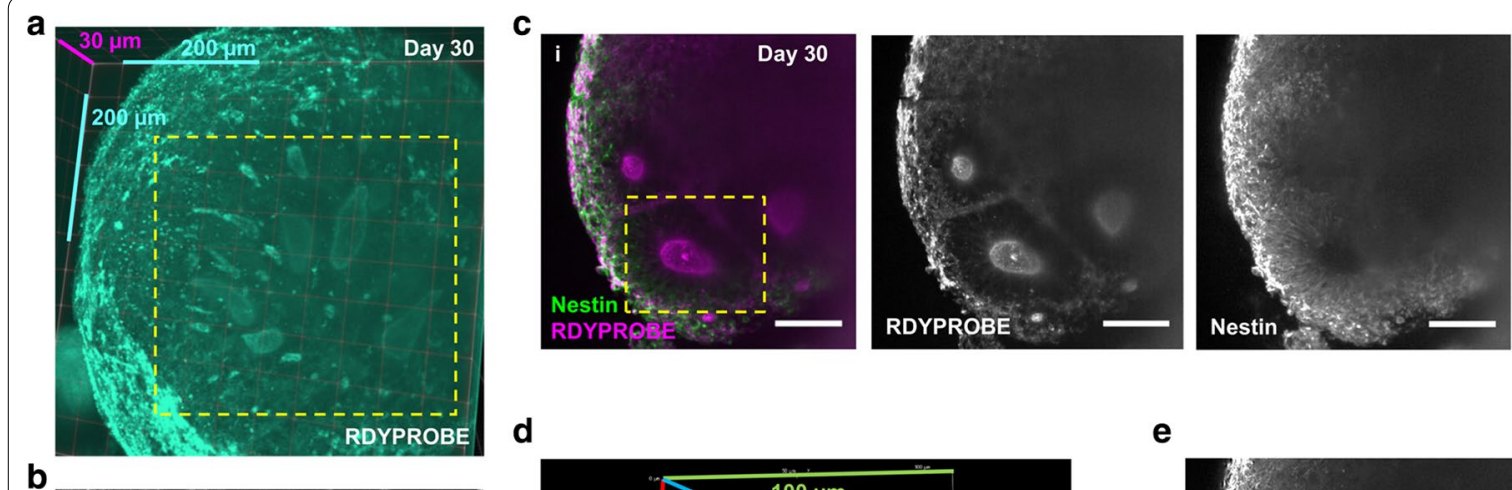

\section{d}
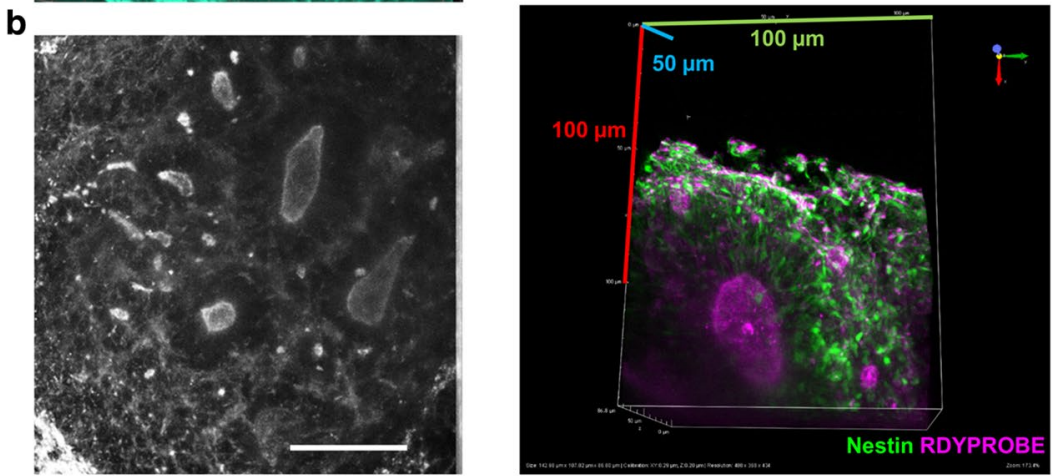

e

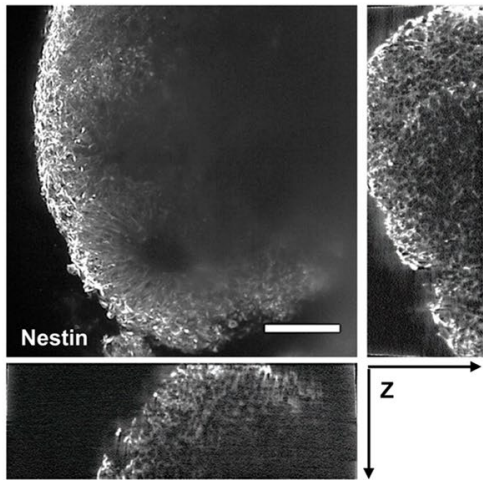

Fig. 4 Visualising internal structures of hCSs using Airy-beam light sheet fluorescent microscopy. a Clearvolume 3D representation of multiple internalised rosette lumen of differing sizes shown by RDYPROBE staining (F-actin) with dimensional scale. b Magnified region (a, dotted line) showing characteristic ovoid or tubular shaped lumens expected of a ventricle-like structure. c Composite (i) and individual channel max-projected images of (ii) RDYPROBE (F-actin) staining and (iii) Nestin-positive filaments around apical membranes, showing the presence of multiple large rosettes, Scale bars $=100 \mu \mathrm{m}$. d 3D render of magnified region from ci (dotted line) displaying of a singular rosette lumen co-stained for radial Nestin filaments and RDYPROBE (F-actin) with dimensional scale. e Orthogonal views of stained tissues permit examination of a cortical rosette in all axes. Scale bar $=50 \mu \mathrm{m}$

However, examination of individual channels revealed differences in signal intensity and image clarity at depth (Fig. 4c-e). F-actin staining could be seen though most of the spheroid (Fig. 4c, d; Additional file 1: Figure 4D), with signal intensity dropping when reaching the inner most regions of the spheroid. Similarly, the signal intensity from Nestin staining substantially dropped at more superficial depths compared to the F-actin stain, with a loss of resolution at depth towards the centre of the imaged hCS (Fig. 4d, e). Thus, visualisation of the colocalisation of Nestin-positive cells and F-actin associated filaments around the apical domain of the rosette lumen was limited at depth $(>200 \mu \mathrm{m})$. Despite this, orthogonal examination displayed ALSM maintains a much greater resolution and clarity compared to confocal when imaging at depth (Fig. 4e). Together, these data demonstrate that the ALSM can rapidly acquire multichannel 3D volumes, but with limited resolution when imaging hCS at depth.

\section{Optimised immunohistochemistry and tissue clearing improves ALSM imaging of deep structures within hCS} Studies have shown that effective imaging at depth can be enhanced by clearing the 3D tissue and optimising immunostaining protocols before LSM imaging [25, 38, 39]. As our day 30 spheroids were approximately $2 \mathrm{~mm}$ in diameter (Additional file 1: Figure 1E), we reasoned that optimizing our immunostaining protocol would improve antibody penetration in 3D tissue as previously described [32]. As, it is well understood that lipids cause light scattering which interfere with deep tissue imaging, we further reasoned that cleared our hCSs so that the lipids were replaced with a clear water-soluble matrix, would reduce light scattering and therefore improve fluorescent signal within spheroids [31, 32]. Optimised immunostaining was achieved by using glycine and DMSO at low concentrations which improve $\mathrm{pH}$ and antibody stability for longer incubation periods, while using Tween20 to help make cells more porous for better antibody penetration [32]. Next, hCSs underwent tissue clearing using the ScaleS4 method, a rapid aqueous-based tissue clearing approach that which takes $4 \mathrm{~h}$ [31]. 
(See figure on next page.)

Fig. 5 ALSM imaging of cleared hCSs. a Representative 3D volume image $(600 \times 600 \times 600 \mu \mathrm{m})$ of cleared day 30 hCS immunostained with ZO-1 (green) and Sox2 (magenta) and imaged by ALSM. b Schematic image of ALSM optical sections of cleared hCS shown in c. c ALSM optical Sects. $(600 \times 600 \times 50 \mu \mathrm{m})$ at 450, 500 and $550 \mu \mathrm{m}$ depth, of cleared hCS shown in a. Cyan arrows indicate ZO-1-positive rosette lumen with surrounding radially organised Sox2-positive NPCs. Green and red bars indicate scale in X and Y planes (600 $\mu \mathrm{m})$ —blue scale bar indicates scale in Z plane $(600 \mu \mathrm{m}$ in $\mathbf{a}$ and $50 \mu \mathrm{m}$ in c)

Cleared hCSs were immunostained with Sox2 (NPC marker) and ZO-1 as a marker of the apical membrane of rosettes (Additional file 1: Figure 5A). Unlike non-cleared hCSs, antibody signal could be detected throughout the $600 \times 600 \times 600 \mu \mathrm{m}$ captured image without any obvious loss of fluorescent signal (Fig. 5a-c; Additional file 1: Figure 5B and Additional file 2: Movie 1). Both ZO-1 positive apical membranes as well as Sox2-positive cells could be easily detected in optical sections at various levels within the 1501 image z-stack (Additional file 1: Figure 5B). Each image was acquired with a $50 \mathrm{~ms}$ exposure time; thus, the acquisition of a 1501 slice stack took $75 \mathrm{~s}$ per channel. It is of note that several 'cones' of bright signal could be observed at the peripheral of the images (Fig. 5a; white asterisks). This is likely due to saturation of the detector due to fluorophore crystal/condensates creating artefacts after deconvolution. In 3D renders of the imaged volume (Fig. 5b), both ZO-1 and Sox 2 could be seen at different depth within the spheroid (Fig. $5 \mathrm{c}$ and Additional file 2: Video 1). Consistent with expected results, Sox2-positive cells were organised in a radial manner around ZO-1 positive rosette lumens (Fig. 5c; Additional file 1: Figure 5B). Together these data indicate that the combination of an optimised immunostaining protocol with an aqueous clearing method vastly improves imaging depth in hCS.

\section{ALSM imaging of hCS reveals 3D morphology of neural rosette inner lumen}

Confocal imaging of sectioned day $17 \mathrm{hCS}$ indicated that the inner lumen of neural rosettes may develop large spherical or tubular lumens (Fig. 3c-e). However, it was not possible to gain an understanding of the 3D morphology of the central lumen or surrounding cells by confocal microscopy due to signal-to-noise limitations at greater depths $(>100 \mu \mathrm{m})$ (Additional file 1: Figure 2). Conversely, ALSM imaging of day $30 \mathrm{hCS}$ stained with ZO-1 (Fig. 5) indicated that it may be possible to visualise the intact inner lumen of a rosette. To this end, we generated 3D renders of the ZO-1 channel from ALSM imaged day 30 cleared hCSs. Multiple ZO-1 positive structures could be detected within a single hCS (Fig. 6a). Morphological measurements of ZO-1-positive rosette lumens revealed that they varied in size and volume (Fig. 6a, b) - only structures with a volume of $>150 \mu \mathrm{m}^{3}$ were considered as true rosette lumens [16] and included in morphological analyses (Fig. 6c-f). Most ZO-1-positive lumens (>95\%) had volumes ranging between $\sim 150$ to $<10,000 \mu^{3}: 75 \%$ of measured lumens were less than $1000 \mu^{3}$ (Fig. 6c), and a surface area that ranged between $\sim 200$ to $>10,000 \mu^{2}$; again the majority of lumens $(\sim 66 \%)$ were less than $1000 \mu^{2}$ (Fig. 6d). A single large rosette lumen could be readily observed (Fig. 6g, h; Additional file 1: Figure 6A \& B). This structure had volume of 192,484.21 $\mu \mathrm{m}^{3}$ and corresponding surface area of $146,697.61 \mu \mathrm{m}^{2}$. It was also possible to extract additional morphological parameters including length at the longest axis and shape factor, a measure of sphericity (Fig. 6e, f). Finally, by focusing on a single large rosette lumen, it was possible to gain an appreciation of the radial organisation of Sox2-positive NPCs surrounding the inner lumen of rosettes in 3D (Fig. 6i; Additional file 1: Figure 6C and Additional file 3: Movie 2). Taken together, these data demonstrate that following an optimised immunohistochemical and tissue clearing approach, that it is possible to gain insights into the 3D structure and organisation of neural rosettes and surrounding NPCs in hCSs.

\section{Discussion}

The ability to generate 3D brain organoids from somatic cell-derived human hiPSC is a great tool to further our understanding of human brain development in health and disease $[2-4,6,7,17]$. Multiple lines of evidence indicate prenatal development as a critical period for the emergence of cellular differences that may contribute to the emergence of autism [8]. Studies using patient derived hiPSCs grown as either $2 \mathrm{D}$ adherent cultures or as $3 \mathrm{D}$ organoids have further supported such findings [9-12]. To date, most studies utilizing 3D organoids have relied on conventional tissue processing and imaging modalities that are not optimized for the preservation and imaging of intact organoids [25, 28]. This result in the loss of the significance and understanding of the cellular processes, organisation and complexity within a three-dimensional environment [25]. Here, we have optimized an immunohistochemical and tissue clearing approach in combination with ALSM imaging that enables rapid and high resolution imaging of intact hCS. Using this approach, we were able to visualise and quantify the three-dimensional cellular structure of the inner lumen of neural rosettes. This structure is described as an in vitro correlate of the human neural tube which begins to form at gestational week 4 and is the source of the entire central nervous system [16]. As the formation or maintenance of structure of neural rosettes has been reported to be altered 
a

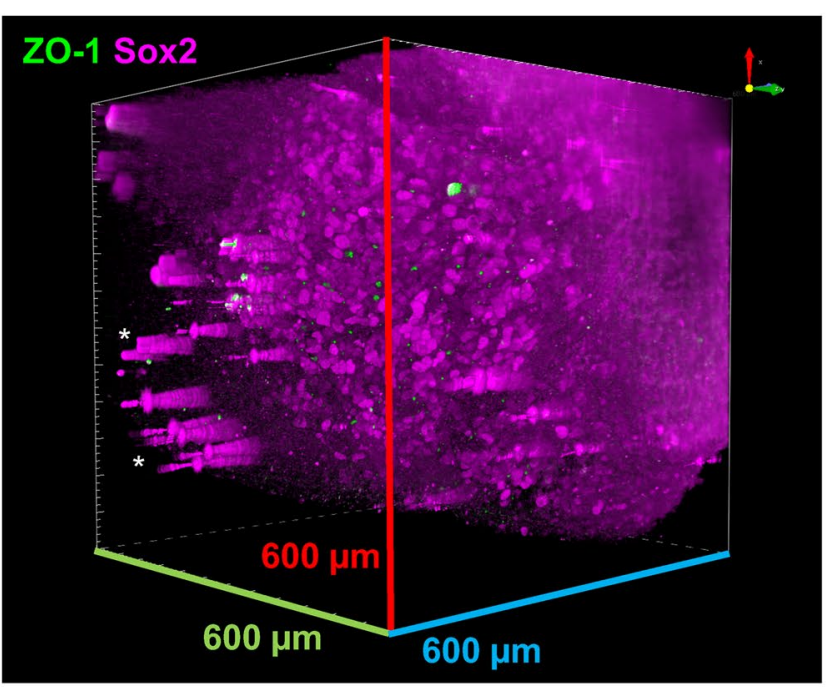

c
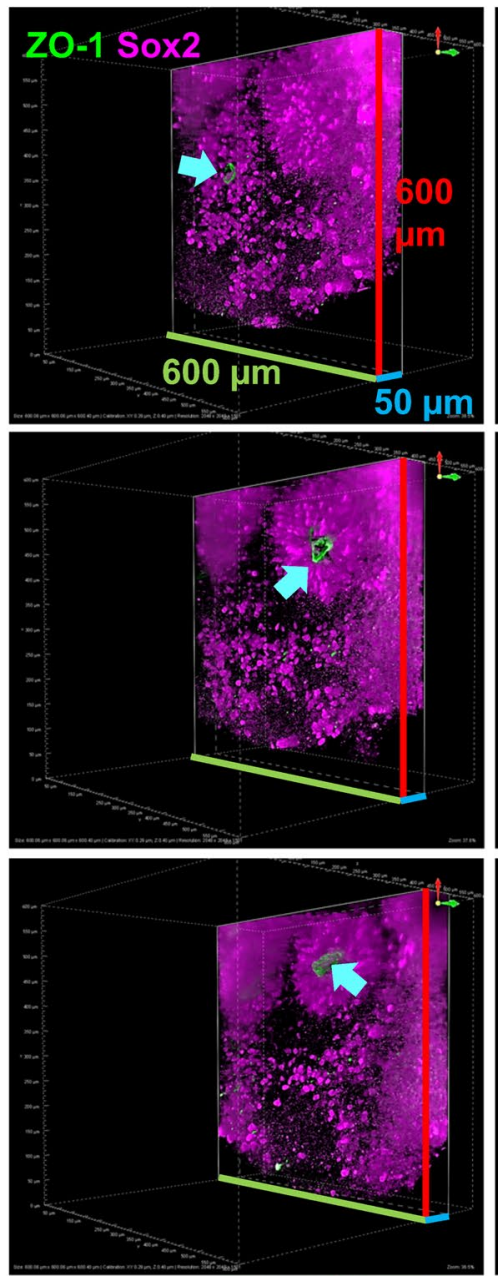

b

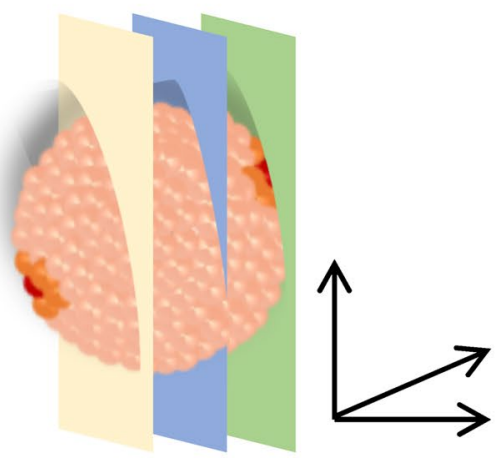

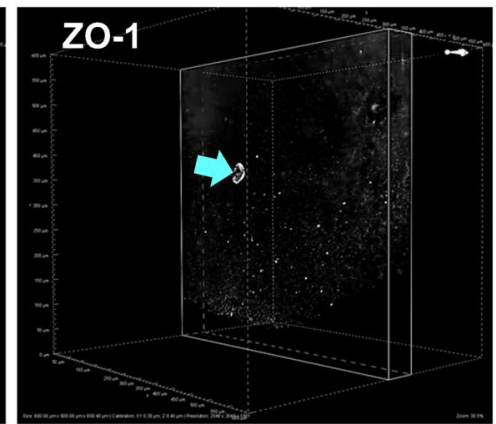
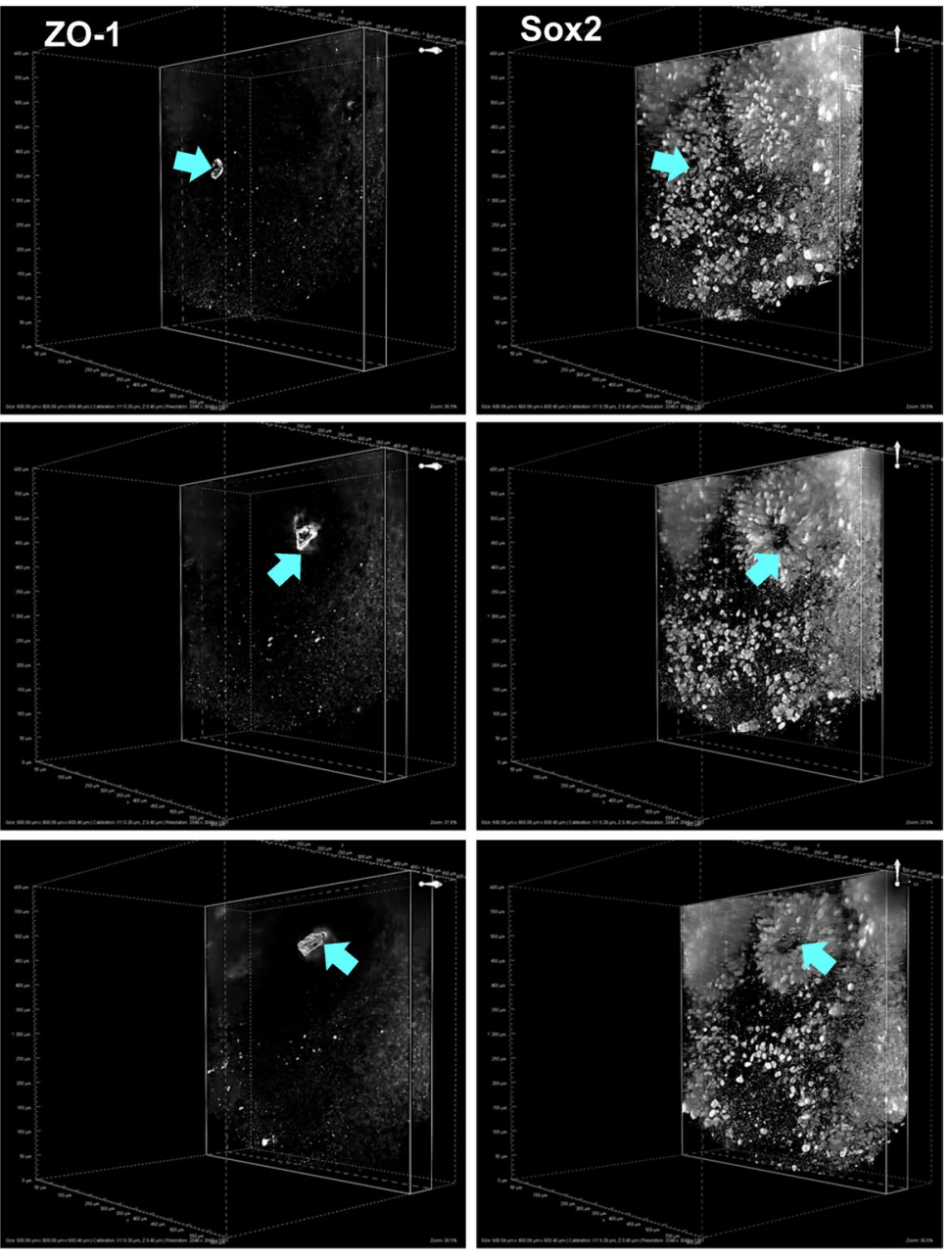

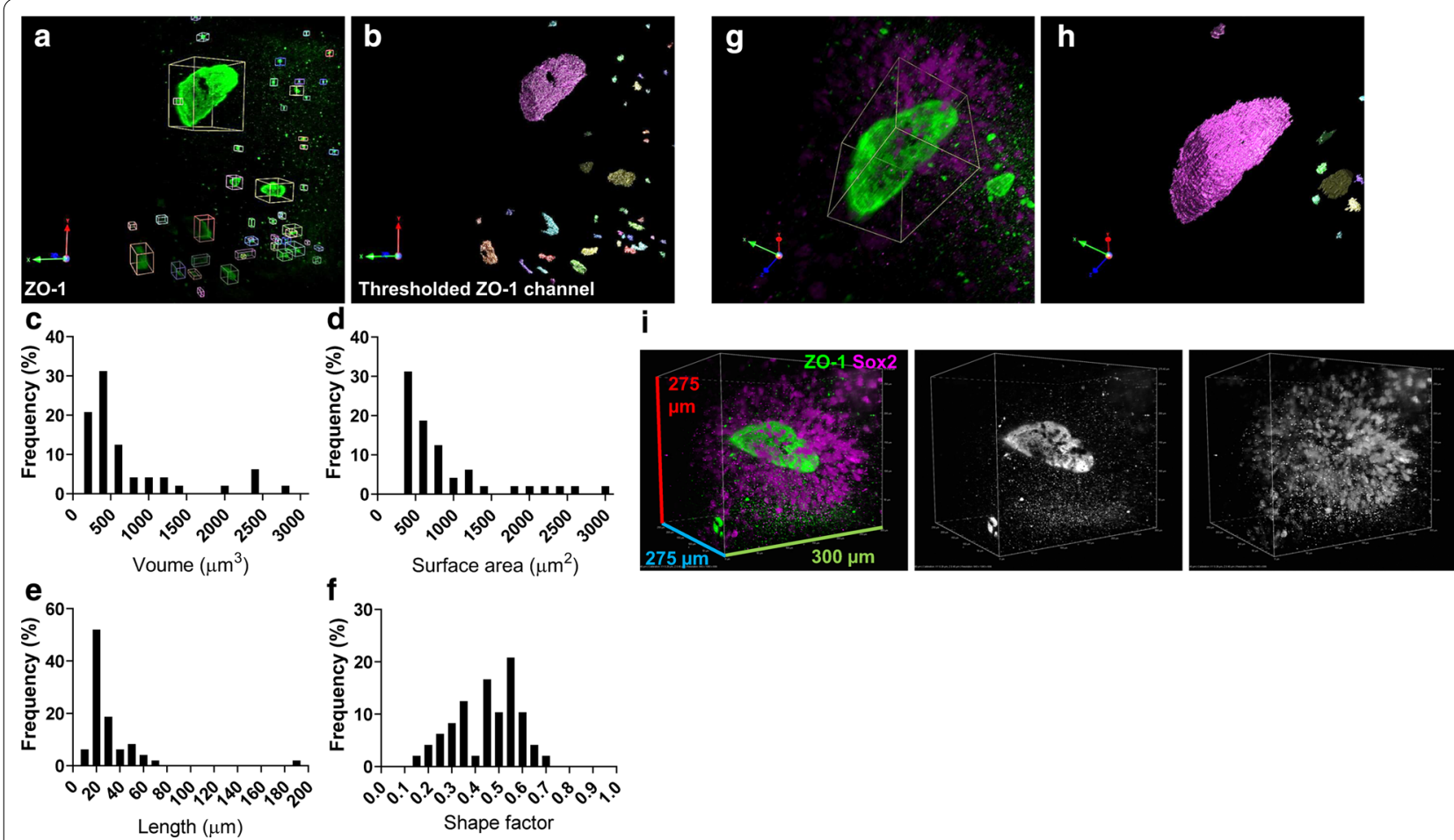

Fig. 6 Visualisation and quantification of neural rosette lumen morphology in intact cleared hCSs. a Representative 3D volume images of ZO-1-positive apical lumen membranes of cleared day 30 hCS. b Rosette lumens were defined as objects in Volocity (PerkinElmer) by thresholding volumetric regions based on ZO-1 channel. Objects with volumes of less than $150 \mu^{3}{ }^{3}$ were discarded. $\mathbf{c}$, d Frequency distribution of neural rosette lumen volume $\left(\mu \mathrm{m}^{3} ; \mathbf{c}\right)$ and surface area $\left(\mu \mathrm{m}^{2} ; \mathbf{d}\right)$. Measurements of these morphological parameters revealed a single neural rosette with a lumen with a volume of $192,484.21 \mathrm{~mm}^{3}$ and surface area of $146,697.61 \mu^{2}$. e, f frequency distribution of neural rosette lumen length ( $\mu \mathrm{m} ; \mathbf{e}$ ) and shape factor $\mathbf{f}, \mathbf{g}$ Digital zoom of largest neural rosette lumen. Green channel indicates ZO-1-positive apical membrane; magenta channel indicates Sox2-positive NPCs surrounding central lumen. $\mathbf{h}$ Threshold image of ZO-1-positive lumen demonstrating morphology of structure in 3D. i Representative 3D render of largest neural rosette lumen demonstrating the radial organisation of Sox2-positive NPCs surrounding ZO-1-positive (green) rosette lumen

in autism and related neurodevelopmental disorders $[8$, $12,40]$, being able to examine the cellular organisation and morphology of neural rosettes in 3D may offer insight into pathological mechanisms that contribute to the likelihood of developing such disorders.

Conventional methods to study brain organoids such as hCS are often hampered by a number of limitations such as the inability to acquire images of cellular structures and organisation within intact specimens. For example, we found that even imaging of intact hCS with a diameter of $\sim 300 \mu \mathrm{m}$ resulted in a rapid loss of fluorescence signal approximately $100 \mu \mathrm{m}$ into the tissue. This was not due to an issue of antibody penetration as the observed signal loss was also observed when imaging DAPI, a nonantibody stain. We were able to overcome this limitation by using an ALSM imaging modality coupled with optical tissue clearing and optimized immunohistochemistry. Advantages of using this form of LSM include the ability to rapidly acquire images in a large volume and a large FOV [27]. This allowed visualization of the inner lumen of neural rosettes in 3D in a manner not possible using confocal microscopy. Using the F-actin stain RDYPROBE, we observed ovoid- or tubular-shaped lumens of neural rosettes. It was also possible to observed radial arrangement of NPCs cells around the lumens. This was our first evidence of the 3D shape of neural rosettes and surrounding NPCs.

Despite this improved ability to image neural rosettes within intact hCSs, a loss of signal was still evident at depth, albeit much less that than observed following confocal imaging. It is well recognised that the presence of lipids within tissue samples causes a scattering of light and therefore impedes effective volume imaging [25, 29, $31,38]$. In addition, uneven, or incomplete penetrance of antibodies could also contribute to this issue [32]. Our approach to address the issue of uneven or incomplete antibody penetration was to use the optimized immunostaining approach described in the FACT clearing protocol [32]. However, in this protocol, tissue clearing takes 1 week followed by 4 days for antibody staining. 
In order to shorten this duration, we used the optimised immunostaining protocol in FACT (4 days) in conjunction with a more efficient and quick clearing method, ScaleS4 which takes $4 \mathrm{~h}$ [31]. By combining this approach with ScaleS4 clearing, our tissue preparation time was reduced to less than half ( $\sim 5$ days) of the FACT protocol. This is also much less compared to other established protocols such as CLARITY ( 20 days) and CUBIC ( 11 days) $[29,31,32]$. As FACT and ScaleS4 were both aqueous-based protocols, we found they were quite compatible when used together. This optimized tissue clearing approach further improved ALSM imaging of intact $\mathrm{hCS}$. This allowed us to visualise the inner lumen and the radial organisation of the NPCs surrounding the inner domain of the rosette, and thus providing a representation of the 3D structure of the neural rosette. This is of particular interest as our previous studies indicate that abnormal neural rosette formation is apparent in autism hiPSCs differentiated either in 2D adherent cultures or in 3D hCSs [12] (Additional file 1: Figure 3). Importantly, the observed radial arrangement of NPCs around the inner lumen of rosettes is consistent with the organisation of DAPI-stained cells around a central lumen of intact embryoid bodies generated from hiPSCs and subsequently cleared and imaged using a new single-objective planar-illumination two-photon microscope [28]. A notable difference in the system described by Rakotoson et al. is that their system uses a two-photon laser. Such a microscope is also optimised for fast imaging, affords less photobleaching and permits better depth penetration compared to confocal microscopes [28], similar to the ALSM system utilised in this study. We anticipate that two-photon microscopy in general would enable deeper penetration due to the infra-red wavelengths not being absorbed by the tissue. Of note, we have recently described a two-photon ALSM system that is also optimised for fast two-photon imaging at depth [41]. A comparison of both systems, especially in live specimens where fast imaging is desirable, would be of interest in the future. Nevertheless, to our knowledge, this is one of the first studies to demonstrate the ability to visualise the lumen and arrangement of NPCs surrounding neural rosettes in 3D. From the cleared images of neural rosettes, we were able to clearly delineate the edges of the lumen and create a 3D reconstruction of the shape of the lumen. This allowed us to quantify the size of the lumens and found that most lumens in a given spheroid had volumes less than $1000 \mu \mathrm{m}^{3}$, while only few had volumes larger than $10,000 \mu \mathrm{m}^{3}$.

The presence of a single neural rosette with a large lumen is similar to that previously reported [16]. The formation of a single large rosette is considered an essential feature of normal brain development and critical for establishing typical tissue cytoarchitecture and normal differentiation of neuroepithelial cells during brain development [16]. Consistent with this, it was possible to observe the radial organisation of NPCs around the large rosette in 3D [2, 5-7]. Thus, this approach suggests that it is possible to examine typical cellular maturation observed in neurodevelopment in 3D. Thus, this combined approach would be a good cellular model to study neurodevelopmental conditions such as autism.

\section{Limitations}

The ALSM system used in this study has a working distance of $3.5 \mathrm{~mm}$ due to the physical room between the illumination and detection objectives. Furthermore, while a day 7 hCS could fit fully into the FOV, only a part of the day 30 tissues $(600 \times 600 \times 600 \mu \mathrm{m})$ could be imaged. A number of alternative forms of LSMs have been developed that could be used as an alternative [25, 42]. Despite these limits, a rapid acquisition time ( $75 \mathrm{~s}$ for $1501 \mathrm{z}$-slices per channel) allows for quick repositioning of tissues to new fields of view or different ranges of depth.

In this study, we have generated hCS brain organoids as an in vitro model of cortical development. However, this protocol is variable, often producing organoids of various sizes, and moreover, only generates cells representative of the dorsal forebrain. A number of protocols have been described for the generation of 3D brain organoids with less variability and of different brain regions in addition to the generation of 'fused' organoids that model multiple brain regions [1-3, 22-24]. Such approaches could be used to reduce variability and would facilitate the modelling and examination of cellular organisation, process and interaction between multiple brain regions.

The key problem being faced by the field of volumetric acquisition (including the use of ScaleS or CLARITY) is the need for standardised methods of volumetric quantification, in order to fully extrapolate the vastly powerful data obtained [25, 29, 33, 42]. Due to current popularity of $2 \mathrm{D}$ cell culture and sectioned tissues, many means of quantification disregard the possibility of a third spatial dimension. However, a number of programs, including open-source programs, which include high-through acquisition and automated quantification of 3D structures are being developed [42-44].

\section{Conclusion}

In summary, the combined optimised immunohistochemical, optical tissue clearing and ALSM imaging approach described here represents an alternative method to image intact hCSs compared to conventional tissue processing and imaging modalities. Using this approach, it is possible to visualise cellular structures 
critical to human brain development in the hCS in vitro model in 3D. From imaging 3D spheroids, we have observed the ovoid, tubular structure of the lumens, and the radial arrangement of differentiating neural progenitor cells within the neural rosette. We also observed larger neural rosettes were fewer in number in a spheroid, approaching the singularity that is observed in vivo during neural tube formation. This was evidence that this method could be used to study typical human brain development, and characteristics of neural rosette formation could be used to study brain development in neurodevelopmental conditions such as autism.

\section{Methods}

\section{Study aim and design}

The aims of this study encompassed two core objectives: (1) comparison of conventional tissue processing and image acquisition methods against a novel methodology of presenting intact 3D tissue for volumetric acquisition using an ALSM; and (2) to determine whether clarification of tissue would improve acquisition of neural rosettes deep within hCSs. To this end, hCSs were differentiated from 3 previously well characterise hiPSCs [15]-CTR_M1; CTR_M2 and CTR_M3-up to 2 clones per line were utilized in the study. For all experiments, hCSs were generated from each line (1-2 clones); images presented in figures are representative of structures observed from all lines. Between 2 and 5 independent batches of hCS were generated for each experiment where a batch was defined as an independent differentiation from hiPSCs.

\section{HiPSC maintenance}

HiPSCs from 3 typically developing individuals (CTR M1; CTR_M2 and CTR_M3) were generated as previously described as part of EU-AIMS and STEMBANCC EU IMIs [12, 15]. Ethical approval for the generation of hiPSCs was approved under the PiNDS study (REC: 13/ LO/1218). HiPSCs were grown and maintained in E8 medium (Life Technologies) with E8 supplement (Life Technologies). HiPSCs were passaged by first dissociating attached cells from the plate, by incubating them in Versene (Thermo Fisher) for $4-5$ min in $37{ }^{\circ} \mathrm{C}$ incubator. Versene helps gently lift edges of iPSC colonies, after which it was replaced with E8 and cells were lifted using a cell lifter. Free-floating cells were re-plated onto fresh geltrex-coated (Thermo Fisher) plates. For maintenance, cells were re-plated at $\sim 70 \%$ confluency. For starting a new experiment, cells were re-plated at $~ 100 \%$ confluency.

\section{Generation of human cortical spheroids}

HiPSCs were differentiated into human cortical spheroids (hCS) using methods published by Pasca et al. In this method, dual SMAD inhibition was performed using KOSR media supplemented with $10 \mu \mathrm{M}$ ROCKi and SMAD inhibitors: $5 \mu \mathrm{M}$ dorsomorphin (Tocris) and $10 \mu \mathrm{M}$ SB431542 (Tocris) to direct hiPSCs towards a cortical lineage. Cultures were maintained in $5 \% \mathrm{CO} 2$ at $37^{\circ} \mathrm{C}$ for 48 -h period to promote formation of spheroids. From day 2 until day 4, media changes were performed daily with fresh KOSR media supplemented with $5 \mu \mathrm{M}$ dorsomorphin and $10 \mu \mathrm{M}$ SB431542. From day 5 until day 25 (or when hCSs were harvested), culture media used was B27 minus Vitamin A (Life Technologies) media plus $20 \mathrm{ng} / \mathrm{mL}$ of recombinant human EGF and recombinant human bFGF (both Preprotech). From day 25, B27 minus Vitamin A media with $20 \mathrm{ng} / \mathrm{mL}$ of recombinant human BDNF (Preprotech) and recombinant human NT3 (LifeTechnologies) was used. Media was changed every $24 \mathrm{~h}$ during the first 15 days and once every $48 \mathrm{~h}$ thereafter. Spheroids were harvested at days 7 , 17 or 30 .

\section{Sample preparation for confocal microscopy}

Cortical Spheroids harvested at Day 7 were fixed in $4 \%$ formaldehyde in $4 \%$ sucrose/PBS for 45 min then washed three times with PBS. Once fixed, spheroids were permeabilised in $0.3 \%$ Triton X100/PBS for $30 \mathrm{~min}$, before permeabilisation-blocking for an hour in $2 \%$ NGS in $0.3 \%$ Triton X100/PBS. Subsequently spheroids were incubated in a solution containing $1^{\circ}$ antibodies, 2\% NGS and $0.1 \%$ Triton $\mathrm{X} 100 / \mathrm{PBS}$ at $4{ }^{\circ} \mathrm{C}$ for a 48 -h period. Spheroids were then washed three times in PBS before being incubated overnight in a solution of $2^{\circ}$ antibodies, $2 \%$ NGS and $0.1 \%$ Triton X100/PBS in a lightproof container at $4{ }^{\circ} \mathrm{C}$. Post-incubation spheroids were washed twice in PBS before DAPI stain was applied. Prepared samples were mounted intact onto Superfrost plus slides (VWR, 631-0108P) by suspending in Mowiol mounting media (Sigma Aldrich, 81381) and surrounding with silicon grease (to protect tissue from high pressures) before applying glass coverslips (VWR, 631-1574) and leaving for $48 \mathrm{~h}$ to set (Fig. 6b). Prior to imaging using confocal microscopy, slides were stored at $4{ }^{\circ} \mathrm{C}$ in the dark. Spheroids harvested at Day 17 were fixed in 4\% formaldehyde/ PBS for $1 \mathrm{~h}$, followed by three PBS washes before incubation in $30 \%$ sucrose solution (weight/volume) overnight. Once spheroids had normalised, they were placed into moulds and cryopreserved in OCT or M1 freezing media via snap-freezing on dry ice, before storage at $-80{ }^{\circ} \mathrm{C}$. Cryopreserved tissues were cryosectioned using a Leica CM1850 cryostat at $20 \mu \mathrm{m}, 40 \mu \mathrm{m}$ and $60 \mu \mathrm{m}$ section depth prior to mounting on Superfrost plus slides for 
fluorescent immunohistochemistry. Sample slides were treated via the same process as post-fix 2D cell culture coverslips, before being mounted with Mowiol and a glass coverslip. Prepared slides were stored in darkness at $4{ }^{\circ} \mathrm{C}$, before image acquisition via confocal microscopy. Compared to Day 7, Day 30 spheroids were significantly larger (Fig. 5c and 5e) and thus required longer treatment to ensure penetrance of washes into deep tissue. Day 30 spheroids were fixed in $4 \%$ formaldehyde/PBS for $2 \mathrm{~h}$, followed by rinsing with three PBS washes, before a permeabilisation-blocking step of $10 \%$ NGS in $0.3 \%$ Triton X100/PBS for $1 \mathrm{~h} 30 \mathrm{~min}$. Permeabilised spheroids were incubated for $48 \mathrm{~h}$ at $4{ }^{\circ} \mathrm{C}$ in a $1^{\circ}$ solution, consisting of $1^{\circ}$ antibodies in 10\% NGS and 0.1\% Triton X100/PBS. Subsequently spheroids were washed three times with PBS and incubated in a $2^{\circ}$ solution consisting of $2^{\circ}$ antibodies in $10 \%$ NGS and $0.1 \%$ Triton X100/PBS overnight at $4{ }^{\circ} \mathrm{C}$, protected from light. Stained spheroids were washed thrice in PBS before being transferred to $0.02 \% \mathrm{NaN} 3 /$ PBS for long-term storage to prevent microbial growth and tissue degradation. Once ready for imaging, tissues were immersed in $\sim 50{ }^{\circ} \mathrm{C} 0.1 \%$ low melting point agarose and set onto specialised mounting slides for light sheet acquisition (Fig. 6c). Tissues could be reclaimable by disintegrating the agarose-matrix bead in PBS with time, thus permitting reimaging.

Permeabilised samples were incubated in solution, consisting of primary antibodies (Additional file 1: Table 1). Superfrost plus slides using Prolong Gold mounting media (Thermo Fisher, P36934) were used to reduce photo-bleaching of fluorophores and left in darkness at RT overnight to set, before imaging via confocal microscopy.

\section{Sample preparation for ALSM imaging of intact hCS Non-cleared spheroids}

The hCS were first washed in PBS for $3 \mathrm{~min}$ and then fixed in $4 \%$ formaldehyde in $4 \%$ sucrose-PBS. The fixation time was $45 \mathrm{~min}$ for day 30 spheroids. Once the spheroids were fixed, they were washed again in for $3 \mathrm{~min}$ (3 times) in PBS and stored in sucrose 30\% (weight/ volume) the sucrose sinking improves their preservation. After sucrose sinking, the spheroids were washed three times in PBS for three minutes each time prior to the permeabilisation step. In order to permeabilise the tissue, the antibodies could reach their epitopes within the cell membrane and to prevent non-specific staining, the samples were incubated for $60 \mathrm{~min}$ in permeabilisation-blocking solution of $2 \%$ normal goat serum (NGS) in $0.1 \%$ Triton X100 and PBS. Then, the spheroids were incubated in a solution of permeabilisaton-blocking solution containing the primary antibodies for $48 \mathrm{~h}$.
The samples were washed three times for three minutes in PBS and incubated with permeabilisation-blocking solution containing the secondary antibodies and DAPI (nuclear staining) for two hours and kept in darkness to prevent bleaching of the fluorophores.

\section{Cleared spheroids}

The hCS were first washed in PBS for 3 min and then fixed in $4 \%$ formaldehyde solution in $4 \%$ sucrose-PBS overnight at $4{ }^{\circ} \mathrm{C}$. Fixation solution was then removed and stored in sucrose $30 \%(\mathrm{w} / \mathrm{v})$ overnight at $4{ }^{\circ} \mathrm{C}$. Spheroids were then permeabilised and blocked using a perm/ block solution ( $0.6 \mathrm{M}$ glycine, $0.2 \%$ Triton X-100, $2 \%$ goat serum, 20\% DMSO in PBS) overnight at room temperature (RT). Samples were permeabilised for this length of time in order to facilitate antibody penetrance into large tissues. The samples were washed twice in PBST for $1 \mathrm{~h}$ each time at RT. Primary antibody was made using an 'antibody solution' (0.2\% Tween-20, 5\% DMSO, $2 \%$ goat serum, $0.01 \%$ sodium azide in PBS). Spheroids were incubated in antibody solution for 2 days. After 2 days, spheroids were again washed in PBST for $1 \mathrm{~h}$ each at RT. Secondary antibody solution with added Hoechst (for nuclear stain) was made using the same 'antibody solution' as before. This was incubated similarly for 2 days at RT, protecting from light. Following secondary staining, cells were washed with PBST and stored in PBS before clearing and mounting for ALSM imaging.

\section{Clearing and mounting of intact hCS}

To mount samples a set of homemade sample mounts were created by bonding a $46 \mathrm{~mm}$ square weighing boat (Fisherbrand Cat No. 12942860) to a standard $75 \mathrm{~mm}$ microscopy slide using cyanoacrylate glue (Additional file 1: Figure 7). A silicone rubber plinth was constructed by first casting silicone rubber ( 9 parts reagent to 1 part catalyst) in a flat vessel (petri dish) with a textured base (60 grit sandpaper) to a depth of approximately $5 \mathrm{~mm}$ and left to cure for at least $24 \mathrm{~h}$. Once this had set, a $4 \mathrm{~mm}$ cylinder was punched using a hollow hole punch tool. The cylinder was bonded to the middle of the weighing boat with the textured surface facing upwards using cyanoacrylate glue and left to set for a few hours before use. The silicone rubber cylinder formed the imaging plinth for samples to be positioned on allowing lenses to lower around plinth and sample without collision to base of the weighing boat (Additional file 1: Figure 7).

In order to place whole hCSs on to the imaging plinth, $1.2 \% \mathrm{w} / \mathrm{v}$ agarose in water was melted and a small volume (less than $50 \mu \mathrm{l}$ ) was placed on to the plinth and the spheroid was positioned in the top of the still liquid agarose 
shortly after allowing agarose to solidify around the spheroid and holding it still for imaging.

Mounted spheroids were then cleared using the ScaleSQ5 protocol published by Hamma et al. 2015[31]. In this method, clearing was performed by incubating hCSs into ScaleSQ(5) solution (Additional file 1: Table 2) for $2 \mathrm{~h}$ at $37{ }^{\circ} \mathrm{C}$. This was followed by submersion of the cleared tissue in mounting media ScaleS4(0) (Additional file 1: Table 2) for at least $2 \mathrm{~h}$ at room temperature. The mounting media had a refractive index $(\mathrm{RI})=1.437$.

\section{Confocal microscopy}

A Leica laser scanning confocal microscope (TCS-SP5) was used to image Day 7 and 17 2D cultures, as well as Day 7 whole-mount and Day 17 cryosectioned spheroids. Leica's LAS AF software was used to set up and acquire multiple channels of singular planes and $\mathrm{Z}$ stacks at a resolution of $1024 \times 1024$ px. $405 \mathrm{~nm}$ (DAPI; blue), $488 \mathrm{~nm}$ (green), $561 \mathrm{~nm}$ (red) and $633 \mathrm{~nm}$ (far-red) lasers were used to generate images in colour channels. Smart gain was used to adjust the different channels to a similar intensity to highlight fluorescent staining. 2D cultures were acquired at $63 \times$ magnification in oil immersion (NA 1.4 ), whereas $3 \mathrm{D}$ cultures were imaged at $40 \times$ magnification in oil immersion (NA 1.3) to encompass a wider field of view. A line average of three was used in each channel during point scanning to reduce appearances of random background signal caused by light scattering, therefore increasing the quality of acquisition.

\section{Lightsheet microscopy}

An upright Aurora ${ }^{\mathrm{TM}} \mathrm{M}$ Squared single photon Airy beam light-sheet microscope [27] was used to perform the light-sheet imaging on the organoid samples. The fluorophore excitation was delivered with a $10 \times$ water immersion illumination objective (Olympus UMPLNFLN $10 \times$ NA 0.30 ), and emission was captured orthogonal to the excitation plane using a $20 \times$ water immersion detection objective (Olympus UMPLNFN $20 \times$ NA 0.50) to provide a lateral resolution of $670 \mathrm{~nm}$. The emission light passed through a filter wheel before images were captured using a sCMOS camera (Orca Flash 4.0 v2) with a FOV of $600 \mu \mathrm{m}$. Three-dimensional volumes were acquired in step sizes of $0.4 \mu \mathrm{m}$ by XZ translation of the sample through the focal plane and outputted stacks were deconvoluted to provide an axial resolution of $\sim 870 \mathrm{~nm}$. Samples were excited using a $405 \mathrm{~nm}$ (DAPI; blue), $488 \mathrm{~nm}$ (green), $561 \mathrm{~nm}$ (red) and $640 \mathrm{~nm}$ (far-red) lasers and camera exposure rate adjusted to generate optimal $\mathrm{S} / \mathrm{N}$ for multi-colour images. Objective working distance was $3.5 \mathrm{~mm}$ which allowed ample space for positioning of samples.

\section{Image acquisition with lightsheet microscopy Preparation and collection of PSF for deconvolution/image processing with tetraspeck beads}

In order to process the data with the M-Squared Lasers Deconvolution software, a volume of data containing fluorescent microspheres was collected with identical optical and spatial parameters (wavelengths for excitation and emission as well as xy pixel size and $\mathrm{z}$ step size) as the collected specimen data to be able to produce a calibrated point-spread function (PSF). To produce a suspension of fluorescent microspheres adequate for this purpose, $1.2 \mu \mathrm{l}$ of stock solution of $0.5 \mu \mathrm{m}$ fluorescent microspheres (Invitrogen Tetraspeck $0.5 \mu \mathrm{m}-$ Cat No. T7281) was added to $100 \mu \mathrm{l}$ of molten agarose $(1.2 \% \mathrm{w} / \mathrm{v})$ and the mixture was completely mixed by pipette action before dispensing. $50 \mu \mathrm{l}$ of the mixture was drawn, and a dome of agarose was placed on a clean sample mount and allowed to solidify before continuing. Mounted bead suspensions were then placed in the corresponding imaging medium (PBS or ScaleS0(4)) and either used immediately (PBS) or left overnight. For samples or beads in ScaleS0(4) at least one further solution change was then made a few hours before imaging to allow solutions to properly equilibrate and allow any residual water from the agarose to be completely removed.

Acquisition of bead fluorescence volumes was performed by positioning the bead preparation in the focal plane of the lenses with the motorised stage, and first checking excitation beam was visible and focussed by halting scanning mirrors to produce a static beam image and observing position and shape of formed image and adjusting focus of excitation and detection objectives so that the correct focussed beam shape was observed. Once this was satisfied, the scanning mirrors were reactivated and images were collected with excitation power and camera integration time sufficient that the peak image intensity of beads was measured to be at least $10 \%$ of the total camera bit depth (16bit-65,535) producing sufficient signal to noise ratio for software to automatically locate beads from the data set. This criterion was met for each combination of excitation wavelength and emission filter combination required to image a sample and a volume of at least $100 \mu \mathrm{m}$ depth of images spaced $0.4 \mu \mathrm{m}$ apart in focus. The stage was translated in the yz axis with a dedicated stage driver for this axis as this was the perpendicular direction to the focus of the detection objective. These data were analysed and processed by software to obtain the PSF of the imaging setup to be used for deconvolution. This generated a unique PSF and calibration file used on each date of data acquisition and used to deconvolve datasets of sample fluorescence. 


\section{Sample image acquisition, deconvolution and presentation} Samples once mounted were positioned in the focus of the objectives, and data were collected by translation of the motorised stage using the yz driver. Laser power and camera integration times were set to provide sufficient contrast and intensity aiming to minimise total integration time while not compromising signal. The raw data were then passed to M-Squared Lasers proprietary deconvolution software to process for deconvolution and removal of Airy pattern in data. Richardson-Lucy deconvolution was processed with at least 100 iterations, and a final datafile is produced for each channel. These were then combined in Nikon Elements (version 5.02) to overlay channels and produce three-dimensional renders as shown in figures. In some instances, Volocity 6.3.1 (PerkinElmer) or ClearVolume (ImageJ plugin-https:// imagej.net/ClearVolume) were used to produce 3D renders which was chosen only for convenience at the time.

Morphological measurements of lumen volume (Fig. 6) were performed using Volocity 6.3.1 (PerkinElmer) where an object is defined by use of the built-in "find-objects" method and selecting an intensity value to set the threshold for volumetric regions based on the channel used. An arbitrary volume of more than $150 \mu \mathrm{m}^{3}$ was chosen from the dataset, and objects smaller than this were discarded.

\section{Supplementary Information}

The online version contains supplementary material available at https://doi. org/10.1186/s13229-021-00413-1.

\section{Additional file 1. Additional Methods, Figures and Tables.}

Additional file 2. Three-dimensional 'fly-through' of ALSM volumetric image of cleared day 30 hCS immunostained for NPC marker Sox2 (magenta) and aplical membrane marker ZO-1 (green).

Additional file 3. Three-dimensional 'fly-through' digitally zoomed onto largest neural rosette lumen of cleared day 30 hCS. HCS were immunostained for NPC marker Sox2 (magenta) and apical membrane marker ZO-1 (green).

\section{Abbreviations}

ALSM: Airy light-sheet microscope; FOV: Field of view; FWHM: Full width at half maximum; hCSs: Human cortical spheroids; hiPSC: Human induced pluripotent stem cell; LSM: Light sheet microscopy; N-cad: N-cadherin; NPC: Neural progenitor cell.

\section{Acknowledgements}

We thank the Wohl Cellular Imaging Centre (WCIC) at the loPPN, King's College, London, for help with microscopy.

\section{Authors' contributions}

DA, GC, JAC, EV-A, JS, NH, MVY and DPS all carried out experiments, data acquisition and analysis; MVY, ACV and DPS provided technical advice; RF provided unique resources; SB-C, ACV and DPS provided funding; DA, GC, JAC, $\mathrm{NH}$ ACV and DPS wrote the manuscript; DPS oversaw the project. All authors read and approved the final manuscript.

\section{Funding}

The study was supported by grants from the European Autism Interventions (EU-AIMS) and AIMS-2-TRIALS: the Innovative Medicines Initiative Joint
Undertaking under grant agreement no. 115300, resources of which are composed of financial contribution from the European Union's Seventh Framework Programme (FP7/2007-2013) and EFPIA companies' in kind contribution (DPS, SB-C); StemBANCC: support from the Innovative Medicines Initiative joint undertaking under grant 115439-2, whose resources are composed of financial contribution from the European Union [FP7/2007-2013] and EFPIA companies' in-kind contribution (DPS); MATRICS: the European Union's Seventh Framework Programme (FP7-HEALTH-603016) (DPS). In addition, funds from the Wellcome Trust ISSF Grant (No. 097819) and the King's Health Partners Research and Development Challenge Fund, a fund administered on behalf of King's Health Partners by Guy's and St Thomas' Charity awarded to DPS; the Brain and Behavior Foundation (formally National Alliance for Research on Schizophrenia and Depression (NARSAD); Grant No. 25957), awarded to DPS, were used to support this study. ACV acknowledges funding support from the Royal Society [RG130610].

\section{Availability of data and materials}

Data sets are available upon reasonable requests.

Ethics approval and consent to participate:

Ethical approval for the generation of hiPSCs was approved under the PiNDS study (REC: 13/LO/1218).

\section{Competing interests}

JS, NH and RF are all (or were) employees of M Squared Life Ltd.

\section{Author details}

${ }^{1}$ Department of Basic and Clinical Neuroscience, Maurice Wohl Clinical Neuroscience Institute, Institute of Psychiatry, Psychology and Neuroscience, King's College London, London, UK. ${ }^{2}$ Autism Research Centre, Department of Psychiatry, University of Cambridge, Cambridge, UK. ${ }^{3}$ MRC Centre for Neurodevelopmental Disorders, King's College London, London, UK. ${ }^{4}$ M Squared Life Ltd., The Surrey Technology Centre, 40 Occam Road, Guildford, UK. ${ }^{5}$ Department of Neuroimaging, Institute of Psychiatry, Psychology and Neuroscience, King's College London, London, UK.

Received: 22 June 2020 Accepted: 8 January 2021

Published online: 22 January 2021

\section{References}

1. Lancaster MA, Renner M, Martin CA, Wenzel D, Bicknell LS, Hurles ME, Homfray T, Penninger JM, Jackson AP, Knoblich JA. Cerebral organoids model human brain development and microcephaly. Nature. 2013;501:373-9.

2. Pasca AM, Sloan SA, Clarke LE, Tian Y, Makinson CD, Huber N, Kim CH, Park JY, O'Rourke NA, Nguyen KD, et al. Functional cortical neurons and astrocytes from human pluripotent stem cells in 3D culture. Nat Methods. 2015;12:671-8.

3. Qian X, Nguyen HN, Song MM, Hadiono C, Ogden SC, Hammack C, Yao B, Hamersky GR, Jacob F, Zhong C, et al. Brain-region-specific organoids using mini-bioreactors for modeling ZIKV exposure. Cell. 2016;165:1238-54.

4. Bershteyn M, Nowakowski TJ, Pollen AA, Di Lullo E, Nene A, WynshawBoris A, Kriegstein AR. Human iPSC-derived cerebral organoids model cellular features of lissencephaly and reveal prolonged mitosis of outer radial glia. Cell Stem Cell. 2017;20(435-449):e434.

5. Mariani J, Simonini MV, Palejev D, Tomasini L, Coppola G, Szekely AM, Horvath TL, Vaccarino FM. Modeling human cortical development in vitro using induced pluripotent stem cells. Proc Natl Acad Sci USA. 2012;109:12770-5.

6. Otani T, Marchetto MC, Gage FH, Simons BD, Livesey FJ. 2D and 3D stem cell models of primate cortical development identify species-specific differences in progenitor behavior contributing to brain size. Cell Stem Cell. 2016;18:467-80.

7. Subramanian L, Bershteyn M, Paredes MF, Kriegstein AR. Dynamic behaviour of human neuroepithelial cells in the developing forebrain. Nat Commun. 2017:8:14167.

8. Courchesne E, Gazestani VH, Lewis NE. Prenatal origins of ASD: the when, what, and how of ASD development. Trends Neurosci. 2020;43:326-42. 
9. Marchetto MC, Belinson H, Tian Y, Freitas BC, Fu C, Vadodaria K, Beltrao-Braga P, Trujillo CA, Mendes APD, Padmanabhan K, et al. Altered proliferation and networks in neural cells derived from idiopathic autistic individuals. Mol Psychiatry. 2017;22:820-35.

10. Mariani J, Coppola G, Zhang P, Abyzov A, Provini L, Tomasini L, Amenduni M, Szekely A, Palejev D, Wilson M, et al. FOXG1-dependent dysregulation of $\mathrm{GABA} / \mathrm{glutamate}$ neuron differentiation in autism spectrum disorders. Cell. 2015;162:375-90.

11. Schafer ST, Paquola ACM, Stern S, Gosselin D, Ku M, Pena M, Kuret TJM, Liyanage M, Mansour AA, Jaeger BN, et al. Pathological priming causes developmental gene network heterochronicity in autistic subject-derived neurons. Nat Neurosci. 2019;22:243-55.

12. Adhya D, Swarup V, Nagy R, Dutan L, Shum C, Valencia-Alarcon EP, Jozwik KM, Mendez MA, Horder J, Loth E, et al. Atypical neurogenesis in induced pluripotent stem cells from autistic individuals. Biol Psychiatry 2020.

13. Chambers SM, Fasano CA, Papapetrou EP, Tomishima M, Sadelain M, Studer L. Highly efficient neural conversion of human ES and iPS cells by dual inhibition of SMAD signaling. Nat Biotechnol. 2009;27:275-80.

14. Shi Y, Kirwan P, Livesey FJ. Directed differentiation of human pluripotent stem cells to cerebral cortex neurons and neural networks. Nat Protoc. 2012;7:1836-46

15. Shum C, Dutan L, Annuario E, Warre-Cornish K, Taylor SE, Taylor RD, Andreae LC, Buckley NJ, Price J, Bhattacharyya S, Srivastava DP. Delta(9)tetrahydrocannabinol and 2-AG decreases neurite outgrowth and differentially affects ERK $1 / 2$ and Akt signaling in hiPSC-derived cortical neurons. Mol Cell Neurosci. 2020;103:103463.

16. Knight GT, Lundin BF, lyer N, Ashton LM, Sethares WA, Willett RM, Ashton RS. Engineering induction of singular neural rosette emergence within hPSC-derived tissues. Elife. 2018;7:e37549.

17. Lancaster MA, Huch M. Disease modelling in human organoids. Dis Model Mech. 2019;12:dmm039347.

18. Hribkova H, Grabiec M, Klemova D, Slaninova I, Sun YM. Calcium signaling mediates five types of cell morphological changes to form neural rosettes. J Cell Sci. 2018;131:1-12.

19. Telias $M$, Segal $M$, Ben-Yosef $D$. Neural differentiation of fragile $X$ human embryonic stem cells reveals abnormal patterns of development despite successful neurogenesis. Dev Biol. 2013;374:32-45.

20. Costa V, Aigner S, Vukcevic M, Sauter E, Behr K, Ebeling M, Dunkley T, Friedlein A, Zoffmann S, Meyer CA, et al. mTORC1 Inhibition corrects neurodevelopmental and synaptic alterations in a human stem cell model of tuberous sclerosis. Cell Rep. 2016;15:86-95.

21. Fiddes IT, Lodewijk GA, Mooring M, Bosworth CM, Ewing AD, Mantalas GL, Novak AM, van den Bout A, Bishara A, Rosenkrantz JL, et al. Human-specific NOTCH2NL genes affect notch signaling and cortical neurogenesis. Cell. 2018;173(1356-1369):e1322.

22. Bagley JA, Reumann D, Bian S, Levi-Strauss J, Knoblich JA. Fused cerebral organoids model interactions between brain regions. Nat Methods. 2017;14:743-51.

23. Birey F, Andersen J, Makinson CD, Islam S, Wei W, Huber N, Fan HC, Metzler KRC, Panagiotakos G, Thom N, et al. Assembly of functionally integrated human forebrain spheroids. Nature. 2017;545:54-9.

24. Xiang Y, Tanaka Y, Patterson B, Kang YJ, Govindaiah G, Roselaar N, Cakir B, Kim KY, Lombroso AP, Hwang SM, et al. Fusion of regionally specified hPSC-derived organoids models human brain development and interneuron migration. Cell Stem Cell. 2017;21(383-398):e387.

25. Rios AC, Clevers H. Imaging organoids: a bright future ahead. Nat Methods. 2018;15:24-6.

26. Stelzer EH. Light-sheet fluorescence microscopy for quantitative biology. Nat Methods. 2015;12:23-6.

27. Vettenburg T, Dalgarno HI, Nylk J, Coll-Llado C, Ferrier DE, Cizmar T, GunnMoore FJ, Dholakia K. Light-sheet microscopy using an Airy beam. Nat Methods. 2014;11:541-4.
28. Rakotoson I, Delhomme B, Djian P, Deeg A, Brunstein M, Seebacher C, Uhl R, Ricard C, Oheim M. Fast 3-D imaging of brain organoids with a new single-objective planar-illumination two-photon microscope. Front Neuroanat. 2019;13:77.

29. Chung K, Deisseroth K. CLARITY for mapping the nervous system. Nat Methods. 2013;10:508-13.

30. Gomez-Gaviro MV, Balaban E, Bocancea D, Lorrio MT, Pompeiano M, Desco M, Ripoll J, Vaquero JJ. Optimized CUBIC protocol for three-dimensional imaging of chicken embryos at single-cell resolution. Development. 2017;144:2092-7.

31. Hama H, Hioki H, Namiki K, Hoshida T, Kurokawa H, Ishidate F, Kaneko T, Akagi T, Saito T, Saido T, Miyawaki A. ScaleS: an optical clearing palette for biological imaging. Nat Neurosci. 2015;18:1518-29.

32. Xu N, Tamadon A, Liu Y, Ma T, Leak RK, Chen J, Gao Y, Feng Y. Fast freeof-acrylamide clearing tissue (FACT) — an optimized new protocol for rapid, high-resolution imaging of three-dimensional brain tissue. Sci Rep. 2017;7:9895.

33. Renner M, Lancaster MA, Bian S, Choi H, Ku T, Peer A, Chung K, Knoblich $J A$. Self-organized developmental patterning and differentiation in cerebral organoids. EMBO J. 2017;36:1316-29.

34. LiY, Muffat J, Omer A, Bosch I, Lancaster MA, Sur M, Gehrke L, Knoblich $J A$, Jaenisch R. Induction of expansion and folding in human cerebral organoids. Cell Stem Cell. 2017;20(385-396):e383.

35. Kadoshima T, Sakaguchi H, Nakano T, Soen M, Ando S, Eiraku M, Sasai Y. Self-organization of axial polarity, inside-out layer pattern, and speciesspecific progenitor dynamics in human ES cell-derived neocortex. Proc Natl Acad Sci USA. 2013;110:20284-9.

36. Lucy LB. An iterative technique for the rectification of observed distributions. Astron J. 1974;79:745.

37. Richardson WH. Bayesian-based iterative method of image restoration. JoSA. 1972;62:55-9.

38. Keller PJ, Dodt HU. Light sheet microscopy of living or cleared specimens. Curr Opin Neurobiol. 2012;22:138-43.

39. Nylk J, McCluskey K, Aggarwal S, Tello JA, Dholakia K. Enhancement of image quality and imaging depth with Airy light-sheet microscopy in cleared and non-cleared neural tissue. Biomed Opt Express. 2016;7:4021-33.

40. Yoon KJ, Nguyen HN, Ursini G, Zhang F, Kim NS, Wen Z, Makri G, Nauen $\mathrm{D}$, Shin JH, Park Y, et al. Modeling a genetic risk for schizophrenia in iPSCS and mice reveals neural stem cell deficits associated with adherens junctions and polarity. Cell Stem Cell. 2014;15:79-91.

41. Hosny NA, Seyforth JA, Spickermann G, Mitchell TJ, Almada P, Chesters R, Mitchell SJ, Chennell G, Vernon AC, Cho K, et al. Planar Airy beam lightsheet for two-photon microscopy. Biomed Opt Express. 2020;1 1:3927-35.

42. Tomer R, Lovett-Barron M, Kauvar I, Andalman A, Burns VM, Sankaran S, Grosenick L, Broxton M, Yang S, Deisseroth K. SPED light sheet microscopy: fast mapping of biological system structure and function. Cell. 2015;163:1796-806.

43. Alsehli H, Mosis F, Thompson C, Hamrud E, Wiseman E, Gentleman E, Danovi D. An integrated pipeline for high-throughput screening and profiling of spheroids using simple live image analysis of frame to frame variations. Methods 2020.

44. McQuin C, Goodman A, Chernyshev V, Kamentsky L, Cimini BA, Karhohs KW, Doan M, Ding L, Rafelski SM, Thirstrup D, et al. Cell Profiler 30: nextgeneration image processing for biology. PLoS Biol. 2018;16:e2005970.

\section{Publisher's Note}

Springer Nature remains neutral with regard to jurisdictional claims in published maps and institutional affiliations. 